\title{
22. RADIOLARIAN BIOSTRATIGRAPHY IN THE CENTRAL INDIAN OCEAN, LEG 115
}

\author{
David A. Johnson ${ }^{2}$
}

\begin{abstract}
Identifiable radiolarians of stratigraphic importance were recovered at eight of the sites drilled on Leg 115 . The assemblages range in age from Holocene to middle Eocene (Dictyoprora mongolfieri Zone, about $48 \mathrm{Ma}$ ). Faunal preservation is particularly good in two stratigraphic intervals: the Holocene through upper Miocene (0-9 Ma), and the lowermost Oligocene to middle Eocene (35-48 Ma). Fluctuating rates of silica accumulation at these drill sites during the Cenozoic reflect changing tectonic and paleoceanographic conditions. In particular, the gradual closure of the Indonesian and Tethyan seaways and the northward migration of the Indian subcontinent severely restricted zonal circulation and silica accumulation in tropical latitudes during the late Oligocene through middle Miocene. By the late Miocene the Indian subcontinent had moved sufficiently north of the equator to allow trans-Indian zonal circulation patterns to become reestablished, and biosiliceous sedimentation resumed.

The composition of the radiolarian assemblages in the tropical Indian Ocean is closely comparable with that of the "stratotype" sequences in the equatorial Pacific. However, there are some notable exceptions in Indian Ocean assemblages: (1) the scarcity of the genera Pterocanium and Spongaster in the Neogene; (2) the absence of the stratigraphically important Podocyrtis lineage, $P$. diamesa $\rightarrow P$. phyxis $\rightarrow P$. ampla, in the middle Eocene; and (3) the scarcity of taxa of the genus Dorcadospyris, with the exception of $D$. ateuchus.

The succession of radiolarian events was tabulated for those stratigraphic intervals where the assemblages were well preserved. We identified 55 events in the middle Eocene to earliest Oligocene, and 31 events in the late Miocene to Holocene. The succession of events is closely comparable with that of the tropical Pacific. However, there are exceptions that appear to be real, rather than artifacts of sample preservation, mixing, and core disturbance.
\end{abstract}

\section{INTRODUCTION AND METHODS}

Radiolarians were recovered in eight of the sites occupied during Ocean Drilling Program (ODP) Leg 115 in the western Indian Ocean. The locations and water depths of these sites appear in Table 1.

The upper portions of several of these sites were double- or triple-cored with the advanced hydraulic piston corer (APC). For purposes of this report, radiolarian biostratigraphy is reported only for a composite stratigraphic section at each site. There was insufficient time available to analyze all cores obtained from the duplicate- or triplicate-cored intervals.

Within those stratigraphic intervals where radiolarian preservation was good, four samples per core were prepared and examined. Ordinarily, these samples were obtained from Sections $2,4,6$, and the core catcher (CC). The sediment was prepared by means of standard techniques (Sanfilippo et al., 1985, p. 633 ) and was sieved at $63 \mu \mathrm{m}$; two strewn slides were prepared for each sample.

For each sample examined, qualitative estimates of radiolarian abundance $(\mathrm{C}=$ common; $\mathrm{F}=$ few $; \mathrm{R}=$ rare; $+=$ very rare; $-=$ absent) and preservation $(\mathrm{G}=$ good; $\mathrm{M}=$ moderate; $\mathrm{P}=$ poor) were made. Raw data tables are included in this report for those sites containing significant stratigraphic intervals with identifiable radiolarians. These tabulations include qualitative assessments of the relative abundance of each taxon in the two strewn slides examined for each sample. The criteria used are:

$\mathrm{C}=$ common $(>5 \%$ of a given assemblage $)$;

$\mathrm{F}=\mathrm{few}(1 \%-5 \%$ of a given assemblage);

\footnotetext{
${ }^{1}$ Duncan, R. A., Backman, J., Peterson, L. C., et al., 1990. Proc. ODP, Sci. Results, 115: College Station, TX (Ocean Drilling Program).

2 Department of Geology and Geophysics, Woods Hole Oceanographic Institution, Woods Hole, MA 02543, U.S.A.
}

Table 1. Location and water depths of sites drilled during Leg 115.

\begin{tabular}{|c|c|c|c|}
\hline Site & Latitude & Longitude & $\begin{array}{c}\text { Water } \\
\text { depth } \\
\text { (m) }\end{array}$ \\
\hline 707 & $07^{\circ} 32.7^{\prime} \mathrm{S}$ & $59^{\circ} 01.0^{\prime} \mathrm{E}$ & 1552 \\
\hline 708 & $05^{\circ} 27.3^{\prime} \mathrm{S}$ & $59^{\circ} 56.6^{\prime} \mathrm{E}$ & 4109 \\
\hline 709 & $03^{\circ} 54.9^{\prime} \mathrm{S}$ & $60^{\circ} 33.1^{\prime} \mathrm{E}$ & 3041 \\
\hline 710 & $04^{\circ} 18.7^{\prime} \mathrm{S}$ & $60^{\circ} 58.8^{\prime} \mathrm{E}$ & 3824 \\
\hline 711 & $02^{\circ} 44.6^{\prime} \mathrm{S}$ & $61^{\circ} 09.8^{\prime} \mathrm{E}$ & 4439 \\
\hline 712 & $04^{\circ} 13.0^{\prime} \mathrm{S}$ & $73^{\circ} 24.4^{\prime} \mathrm{E}$ & 2904 \\
\hline 713 & $04^{\circ} 11.6^{\prime} \mathrm{S}$ & $73^{\circ} 23.7^{\prime} \mathrm{E}$ & 2915 \\
\hline 714 & $05^{\circ} 03.6^{\prime} \mathrm{N}$ & $73^{\circ} 47.2^{\prime} \mathrm{E}$ & 2038 \\
\hline
\end{tabular}

$\mathrm{R}=\operatorname{rare}(0.1 \%-1 \%$ of a given assemblage $)$

$+=$ very rare (only one or two specimens found); and

$-=$ absent.

Unlike some other Deep Sea Drilling Project (DSDP) reports (e.g., Westberg and Riedel, 1982), this chapter does not report quantitative abundances of taxa. Even though for some purposes the qualitative approach adopted here may be less than satisfactory, we think it is sufficient for the primary task of this report, namely, identifying significant radiolarian datum levels and their stratigraphic succession.

A complete listing of species names and taxonomic references for all species examined is presented in Appendix A at the end of this chapter. Abbreviated forms of the species names will be used in the text and in Appendixes B and C.

\section{CENOZOIC RADIOLARIAN ZONATION}

This report adopts the standard Cenozoic radiolarian zonation that has been developed for tropical latitudes (Sanfilippo et al., 1985, pp. 645-648; Nigrini, 1985). In addition, the recent work of Riedel and Sanfilippo is incorporated (see Saunders et al., 1985, pp. 409-413). They have replaced the late Eocene 
Thyrsocyrtis bromia Zone with three zones: the Cryptoprora ornata Zone of Maurasse and Glass (1976), and two new zones, the Calocyclas bandyca Zone and the "Carpocanistrum" azyx Zone. This replacement allows for increased zonal resolution in the late Eocene, an interval in which many radiolarian extinctions and appearances occurred (Saunders et al., 1985; Sanfilippo et al., 1985).

We have chosen not to include in this report the proposed zonal subdivisions of the Pliocene, which have been suggested by Johnson et al. (1989). Even though a revision is clearly needed because of the scarcity of the Spongaster lineage in the Indian Ocean, we have retained here the more familiar zonal names (Sanfilippo et al., 1985) for purposes of designating stratigraphic intervals.

Because of the scarcity of the genus Spongaster in the early Pliocene, the base of the $S$. pentas Zone is approximated by the last occurrence (LO) of Solenosphaera omnitubus. And because of the absence of Podocyrtis ampla in the middle Eocene, the base of the P. ampla Zone is approximated by the LO of Theocotyle conica.

\section{RADIOLARIANS AT EACH SITE}

In this section, the radiolarian findings for each site are summarized. Raw data are presented in Tables 2-6 for those stratigraphic intervals that contain well-preserved assemblages. Using these data tables, listings of radiolarian "events" (first and last appearances) are presented for portions of the Neogene (Appendix B) and Paleogene (Appendix C).

\section{Sites $\mathbf{7 0 5}$ and $\mathbf{7 0 6}$}

Sites 705 and 706, located approximately $5 \mathrm{~km}$ apart, were drilled on the northeastern flank of the Nazareth Bank. All core-catcher samples were examined from these sites, and each sample proved to be barren of radiolarians.

\section{Site 707}

Three holes, all within $0.1 \mathrm{~km}$ of each other, were drilled at Site 707, located on the Mascarene Plateau, at the saddle between the Saya de Malha and Seychelles banks. Table 7 lists the intervals that were cored; all were cored with excellent to good core recovery. Radiolarians were examined only in Holes 707A and $707 \mathrm{C}$ because the sequences obtained in Hole 707B duplicate those in Hole 707A.

\section{Hole 707 A}

Cores $115-707 \mathrm{~A}-1 \mathrm{H}$ through $-3 \mathrm{H}(0-25.8 \mathrm{mbsf})$ contain unidentifiable radiolarian fragments. Cores $115-707 \mathrm{~A}-4 \mathrm{H}$ through $-11 \mathrm{H}(25.8-102.8 \mathrm{mbsf})$ contain moderately to well-preserved assemblages of Pliocene and late Miocene age. The occurrences of taxa and zonal age assignments for this interval are presented in Table 2 .

Cores $115-707 \mathrm{~A}-12 \mathrm{H}$ through $-19 \mathrm{H}(105.3-173.7 \mathrm{mbsf})$ are barren of identifiable radiolarians.

Samples 115-707A-20X-1, $101 \mathrm{~cm}$, through 115-707A-23X-2, $101 \mathrm{~cm}$ (174.7-205.2 mbsf) contain moderately preserved radiolarians diagnostic of the Theocyrtis tuberosa Zone of early Oligocene age. Diagnostic taxa include Lithocyclia angusta, Tristylospyris triceros, Theocyrtis tuberosa, Artophormis gracilis, Didymocyrtis prismatica, Dictyoprora pirum, and Cryptoprora ornata.

Sample 115-707A-23X-4, $101 \mathrm{~cm}$ (208.2 mbsf), contains wellpreserved radiolarians diagnostic of the Cryptoprora ornata Zone of late Eocene age. Key taxa include Lithocyclia aristotelis, Dictyoprora mongolfieri, Cryptoprora ornata, Sethochytris babylo-

Table 2. Radiolarians from Hole 707A.

\begin{tabular}{|c|c|c|c|c|c|c|c|c|c|c|c|c|c|c|c|c|c|c|c|c|c|c|c|c|}
\hline $\begin{array}{l}\text { Core, section, } \\
\text { interval }(\mathrm{cm})\end{array}$ & $\begin{array}{l}\text { Radiolarian } \\
\text { zones }\end{array}$ & 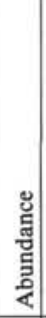 & 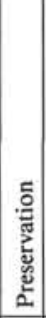 & 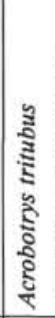 & 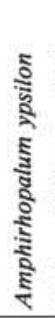 & 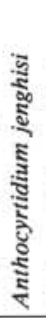 & 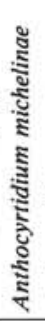 & 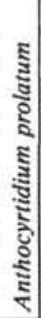 & 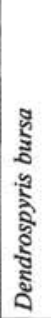 & 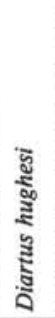 & 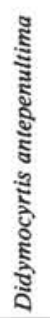 & 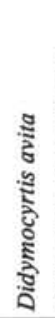 & 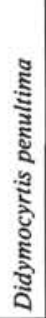 & 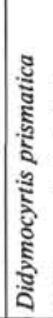 & 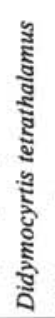 & 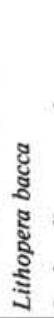 & 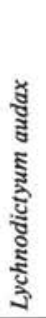 & 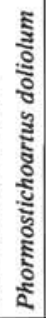 & 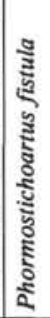 & 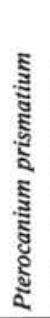 & 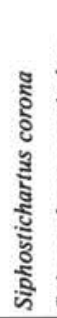 & 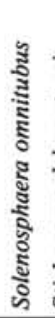 & 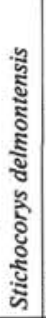 & 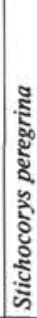 \\
\hline $\begin{array}{l}115-707 \mathrm{~A}-4 \mathrm{H}-2,101-103 \\
115-707 \mathrm{~A}-4 \mathrm{H}-4,101-103 \\
115-707 \mathrm{~A}-4 \mathrm{H}-\mathrm{CC} \\
115-707 \mathrm{~A}-5 \mathrm{H}-2,102-104 \\
115-707 \mathrm{~A}-5 \mathrm{H}-4,101-103 \\
115-707 \mathrm{~A}-5 \mathrm{H}-5,101-103 \\
115-707 \mathrm{~A}-5 \mathrm{H}-\mathrm{CC} \\
115-707 \mathrm{~A}-6 \mathrm{H}-2,101-103 \\
115-707 \mathrm{~A}-6 \mathrm{H}-4,101-103 \\
115-707 \mathrm{~A}-6 \mathrm{H}-6,101-103\end{array}$ & $\begin{array}{l}\text { Spongaster } \\
\text { pentas }\end{array}$ & $\begin{array}{l}\text { R } \\
C \\
F \\
R \\
F \\
C \\
F \\
C \\
C \\
C\end{array}$ & $\begin{array}{l}\mathrm{P} \\
\mathrm{M} \\
\mathrm{M} \\
\mathrm{M} \\
\mathrm{M} \\
\mathrm{M} \\
\mathrm{M} \\
\mathrm{M} \\
\mathrm{G} \\
\mathrm{G}\end{array}$ & & $\begin{array}{l}- \\
- \\
- \\
- \\
- \\
-\end{array}$ & $\begin{array}{l}\mathrm{R} \\
\mathrm{R} \\
\mathrm{R} \\
\mathrm{R} \\
\mathrm{F} \\
\mathrm{R} \\
\mathrm{R} \\
\mathrm{R} \\
\mathrm{F}\end{array}$ & $\begin{array}{l}\mathrm{R} \\
\mathrm{F} \\
\mathrm{F} \\
\mathrm{R} \\
\mathrm{R}\end{array}$ & $\begin{array}{l}\mathbf{R} \\
\mathbf{R} \\
\mathbf{R} \\
\mathbf{R} \\
\mathbf{R} \\
\mathbf{R}\end{array}$ & $\mathbf{R}$ & & & $\begin{array}{l}\bar{R} \\
\mathrm{R} \\
\mathrm{R} \\
\mathrm{R} \\
\mathrm{R} \\
\mathrm{F} \\
\mathrm{F} \\
\mathrm{R} \\
\mathrm{F}\end{array}$ & $\begin{array}{l}- \\
- \\
\bar{R}\end{array}$ & & $\begin{array}{l}\mathrm{F} \\
\mathrm{R} \\
\mathrm{F} \\
\mathrm{R} \\
\mathrm{R} \\
\mathrm{R} \\
\mathrm{R} \\
- \\
-\end{array}$ & $\begin{array}{l}\mathrm{R} \\
\mathrm{R} \\
\mathrm{R} \\
\mathrm{R} \\
\mathrm{F} \\
\mathrm{F} \\
\mathrm{R} \\
\mathrm{F} \\
\mathrm{R} \\
\mathrm{C}\end{array}$ & $\begin{array}{l}\mathrm{R} \\
\mathrm{R} \\
\mathrm{R} \\
\mathrm{R} \\
\mathrm{F} \\
\mathrm{F} \\
\mathrm{F} \\
\mathrm{F} \\
\mathrm{C} \\
\mathrm{F}\end{array}$ & $\begin{array}{c}- \\
\mathrm{R} \\
\mathrm{F} \\
\mathrm{R} \\
\mathrm{F} \\
\mathrm{F} \\
\mathrm{F} \\
\mathrm{R} \\
\mathrm{C} \\
\mathrm{F}\end{array}$ & $\begin{array}{l}+ \\
\mathrm{R} \\
\mathrm{R} \\
\mathrm{R} \\
\mathrm{R} \\
\mathrm{R} \\
\mathrm{R} \\
\mathrm{R} \\
\mathrm{R} \\
\mathrm{R}\end{array}$ & $\begin{array}{l}R \\
R \\
R \\
R \\
R \\
R \\
R \\
R \\
R \\
-\end{array}$ & & $\begin{array}{l}- \\
- \\
- \\
- \\
- \\
- \\
- \\
-\end{array}$ & & $\begin{array}{l}\mathrm{R} \\
\mathrm{C} \\
\mathrm{F} \\
\mathrm{R} \\
\mathrm{C} \\
\mathrm{C} \\
\mathrm{F} \\
\mathrm{F} \\
\mathrm{C} \\
\mathrm{C}\end{array}$ \\
\hline $\begin{array}{l}115-707 \mathrm{~A}-6 \mathrm{H}-\mathrm{CC} \\
115-707 \mathrm{~A}-7 \mathrm{H}-2,101-103 \\
115-707 \mathrm{~A}-7 \mathrm{H}-4,101-103 \\
115-707 \mathrm{~A}-7 \mathrm{H}-6,101-103 \\
115-707 \mathrm{~A}-7 \mathrm{H}-\mathrm{CC} \\
115-707 \mathrm{~A}-8 \mathrm{H}-\mathrm{CC} \\
115-707 \mathrm{~A}-9 \mathrm{H}-2,101-103\end{array}$ & $\begin{array}{l}\text { Stichocorys } \\
\text { peregrina }\end{array}$ & $\begin{array}{l}\mathrm{C} \\
\mathrm{F} \\
\mathrm{C} \\
\mathrm{C} \\
\mathrm{C} \\
\mathrm{F} \\
\mathrm{C}\end{array}$ & \begin{tabular}{l|}
$\mathrm{G}$ \\
$\mathrm{M}$ \\
$\mathrm{M}$ \\
$\mathrm{G}$ \\
$\mathrm{G}$ \\
$\mathrm{M}$ \\
$\mathrm{M}$
\end{tabular} & R & & $\begin{array}{l}\mathrm{R} \\
\mathrm{F}\end{array}$ & $\begin{array}{l}\text { F } \\
\text { R } \\
\text { F } \\
\text { R } \\
\text { R } \\
\text { F } \\
\text { F }\end{array}$ & & $\begin{array}{l}\mathrm{R} \\
\mathrm{R}\end{array}$ & & & $\begin{array}{l}\mathrm{F} \\
\mathrm{F} \\
\mathrm{F} \\
\mathrm{F} \\
\mathrm{R}\end{array}$ & $\begin{array}{l}\mathrm{R} \\
\mathrm{R} \\
\mathrm{R} \\
\mathrm{R} \\
\mathrm{R} \\
\mathrm{R} \\
\mathrm{F}\end{array}$ & & - & $\begin{array}{l}\mathrm{F} \\
\mathrm{R} \\
\mathrm{F} \\
\mathrm{C} \\
\mathrm{R} \\
\mathrm{R} \\
\mathrm{F}\end{array}$ & $\begin{array}{l}\mathrm{F} \\
\mathrm{F} \\
\mathrm{C} \\
\mathrm{C} \\
\mathrm{C} \\
\mathrm{F} \\
\mathrm{F}\end{array}$ & $\begin{array}{l}\mathrm{C} \\
\mathrm{F} \\
\mathrm{F} \\
\mathrm{F} \\
\mathrm{C} \\
\mathrm{F} \\
\mathrm{R}\end{array}$ & $\begin{array}{l}\mathrm{R} \\
\mathrm{R} \\
\mathrm{R} \\
\mathrm{R} \\
\mathrm{R}\end{array}$ & $\begin{array}{l}- \\
- \\
+ \\
- \\
-\end{array}$ & - & $\begin{array}{l}\mathrm{R} \\
\mathrm{R} \\
\mathrm{F} \\
\mathrm{F} \\
\mathrm{R} \\
\mathrm{R} \\
\mathrm{R}\end{array}$ & $\begin{array}{l}- \\
- \\
- \\
- \\
\bar{R} \\
\mathrm{~F}\end{array}$ & $\begin{array}{l}\text { C } \\
F \\
C \\
C \\
C \\
C \\
C\end{array}$ \\
\hline $\begin{array}{l}115-707 \text { A-9H-4, 101-103 } \\
115-707 \text { A-9H-6, 101-103 } \\
115-707 \text { A-9H-CC }\end{array}$ & $\begin{array}{c}\text { Didymocyrtis } \\
\text { penultima }\end{array}$ & $\begin{array}{l}\mathrm{F} \\
\mathrm{R} \\
\mathrm{C}\end{array}$ & $\begin{array}{l}\mathrm{M} \\
\mathrm{M} \\
\mathrm{M}\end{array}$ & & & $\mathbf{R}$ & $\mathbf{R}$ & & $\begin{array}{l}\mathrm{R} \\
\mathrm{F}\end{array}$ & $\begin{array}{l}- \\
- \\
-\end{array}$ & $\begin{array}{l}- \\
- \\
-\end{array}$ & & $\begin{array}{l}\mathrm{R} \\
\mathrm{R} \\
\mathrm{R}\end{array}$ & R & & $\begin{array}{l}\mathrm{F} \\
\mathbf{R}\end{array}$ & $\begin{array}{l}\mathrm{F} \\
\mathrm{R} \\
\mathrm{F}\end{array}$ & $\mathrm{F}$ & R & & - & $\begin{array}{l}\mathrm{R} \\
- \\
-\end{array}$ & $\begin{array}{l}\mathrm{C} \\
\mathrm{F} \\
\mathrm{F}\end{array}$ & $\begin{array}{l}\text { F } \\
+ \\
+\end{array}$ \\
\hline
\end{tabular}


Table 3. Radiolarians from Hole 709A.

\begin{tabular}{|c|c|c|c|c|c|c|c|c|c|c|c|c|c|c|c|c|c|c|c|c|}
\hline $\begin{array}{l}\text { Core, section, } \\
\text { interval }(\mathrm{cm})\end{array}$ & $\begin{array}{l}\text { Radiolarian } \\
\text { zones }\end{array}$ & & 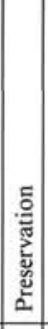 & 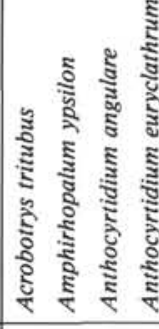 & 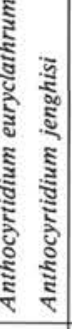 & 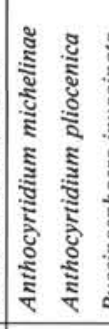 & 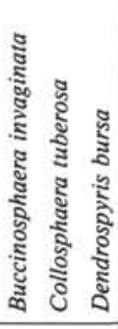 & 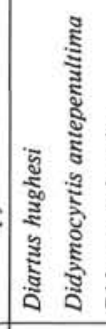 & 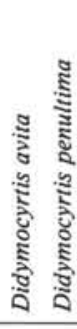 & 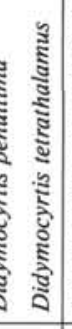 & 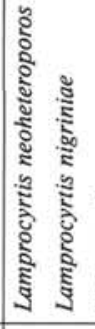 & 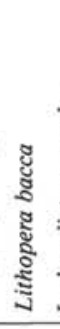 & 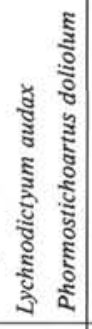 & 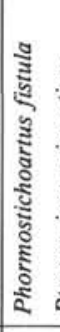 & 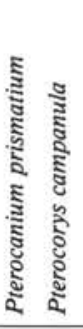 & 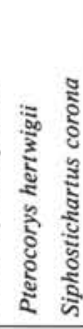 & 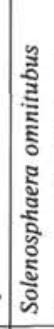 & 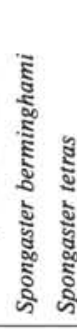 & 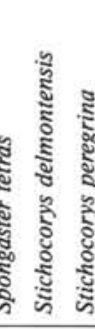 & 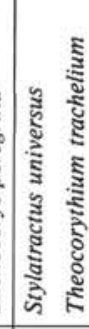 \\
\hline $115-709 \mathrm{~A}-1 \mathrm{H}-2,75-77$ & Buccinosphaera invaginata & C & G & $\mathrm{R}-$ & & & R $\mathbf{R}$ & & & $\mathrm{F}$ & $-F$ & & & & - & R & & $\mathrm{F}$ & $\mathrm{F}$ & $-\mathrm{C}$ \\
\hline $\begin{array}{l}115-709 \mathrm{~A}-1 \mathrm{H}-4,75-77 \\
115-709 \mathrm{~A}-1 \mathrm{H}-\mathrm{CC}\end{array}$ & Amphirhopalum ypsilon & $\begin{array}{l}\text { C } \\
\text { C }\end{array}$ & \begin{tabular}{|l|}
$G$ \\
$G$ \\
\end{tabular} & $\begin{array}{l}\mathrm{F}- \\
\mathrm{C}-\mathrm{R}\end{array}$ & R - & - & -- & & & \begin{tabular}{l|l}
$\mathrm{C}$ \\
$\mathrm{F}$
\end{tabular} & $\begin{array}{l}-\mathrm{R} \\
-\mathrm{R} \\
\end{array}$ & & & & $\overline{-}_{\mathrm{R}}$ & $\underline{\mathrm{R}}$ & & $\mathrm{F}$ & F & $\begin{array}{|ll|}\mathrm{F} & \mathrm{C} \\
\mathrm{R} & \mathrm{C} \\
\end{array}$ \\
\hline $\begin{array}{l}115-709 \mathrm{~A}-2 \mathrm{H}-2,75-77 \\
115-709 \mathrm{~A}-2 \mathrm{H}-4,75-77 \\
115-709 \mathrm{~A}-2 \mathrm{H}-\mathrm{CC}\end{array}$ & Anthocyrtidium angulare & \begin{tabular}{|l|} 
C \\
C \\
C
\end{tabular} & $\begin{array}{l}\mathrm{G} \\
\mathrm{G} \\
\mathrm{G} \\
\end{array}$ & $\begin{array}{lll}\mathrm{C} & \mathrm{R} & \mathrm{R} \\
\mathrm{F} & \mathrm{R} & \\
\mathrm{R} & \mathrm{R} & \\
\end{array}$ & $\begin{array}{r}- \\
- \\
-\end{array}$ & $\overline{-}$ & & & & \begin{tabular}{l|l}
$\mathrm{C}$ \\
$\mathrm{C}$ \\
$\mathrm{F}$
\end{tabular} & $\begin{array}{l}-\bar{R}- \\
\mathrm{F}\end{array}$ & $\begin{array}{l}\mathrm{F} \\
\mathrm{R}\end{array}$ & & & $\begin{array}{ll}- & R \\
- & R \\
- & \\
\end{array}$ & $\overline{-}$ & & $\begin{array}{l}\mathrm{F} \\
\mathrm{F}\end{array}$ & F & $\begin{array}{ll}\mathrm{F} & \mathrm{F} \\
\mathrm{R} & \mathrm{F} \\
\mathrm{C} & \mathrm{F} \\
\end{array}$ \\
\hline $\begin{array}{l}115-709 \mathrm{~A}-3 \mathrm{H}-2,65-70 \\
115-709 \mathrm{~A}-3 \mathrm{H}-2,65-70 \\
115-709 \mathrm{~A}-3 \mathrm{H}-6,65-70\end{array}$ & Pterocanium prismatium & $\begin{array}{l}C \\
C \\
C\end{array}$ & $\begin{array}{l}G \\
G \\
G\end{array}$ & $\begin{array}{l}\mathrm{F}- \\
\mathrm{C}- \\
\mathrm{C}-\end{array}$ & $\begin{array}{l}\bar{R} \\
\mathrm{C}\end{array}$ & $\begin{array}{l}\mathrm{R} \\
\mathrm{R}- \\
\mathrm{R}-\end{array}$ & & & & $\begin{array}{l}\mathrm{R} \\
\mathrm{C} \\
\mathrm{F}\end{array}$ & $\begin{array}{l}\mathrm{F} \\
\mathrm{R}\end{array}$ & $\begin{array}{l}\mathrm{F} \\
\mathrm{F}\end{array}$ & & & $\begin{array}{l}\mathrm{R} \\
\mathrm{R} \\
\mathrm{F}\end{array}$ & & & $\mathrm{F}$ & $F$ & $\begin{array}{ll}\mathrm{C} & \mathrm{F} \\
\mathrm{F} & -\end{array}$ \\
\hline $\begin{array}{l}115-709 \mathrm{~A}-3 \mathrm{H}-\mathrm{CC} \\
115-709 \mathrm{~A}-4 \mathrm{H}-2,65-70 \\
115-709 \mathrm{~A}-4 \mathrm{H}-\mathrm{CC} \\
115-709 \mathrm{~A}-6 \mathrm{H}-2,65-70 \\
115-709 \mathrm{~A}-6 \mathrm{H}-4,65-70 \\
115-709 \mathrm{~A}-6 \mathrm{H}-\mathrm{CC} \\
115-709 \mathrm{~A}-7 \mathrm{H}-4,65-70\end{array}$ & Spongaster pentas & \begin{tabular}{|l|}
$\mathrm{C}$ \\
$\mathrm{F}$ \\
$\mathrm{F}$ \\
$\mathrm{F}$ \\
$\mathrm{F}$ \\
$\mathrm{C}$ \\
$\mathrm{C}$ \\
\end{tabular} & \begin{tabular}{|l|} 
\\
$P$ \\
$P$ \\
$M$ \\
$M$ \\
$G$ \\
$G$ \\
\end{tabular} & $\begin{array}{l}+ \\
\mathrm{R} \\
\mathrm{R} \\
- \\
- \\
-\end{array}$ & $\begin{array}{l}\mathrm{F} \\
\mathrm{R} \\
\mathrm{R} \\
\mathrm{R} \\
\mathrm{R} \\
\mathrm{R} \\
\mathrm{F} \\
\end{array}$ & $\begin{array}{ll}R & - \\
R & + \\
R & + \\
R & R \\
R & R \\
F & R \\
F & R \\
\end{array}$ & & & $\begin{array}{l}\mathrm{R}- \\
\mathrm{F}- \\
\mathrm{F}+\end{array}$ & $\mathrm{F}$ & & R & $\begin{array}{ll}- & - \\
\overline{-} & - \\
\mathrm{F} & \overline{\mathrm{F}} \\
\mathrm{R} & \mathrm{F} \\
\mathrm{R} & \mathrm{F} \\
\mathrm{F} & \mathrm{C} \\
\mathrm{R} & \mathrm{C} \\
\end{array}$ & \begin{tabular}{|l}
- \\
- \\
$R$ \\
$R$ \\
$R$ \\
$F$ \\
$R$ \\
\end{tabular} & $\begin{array}{l}\mathrm{R} \\
\mathrm{R} \\
\end{array}$ & E & $\begin{array}{l}- \\
\overline{-} \\
\overline{-}\end{array}$ & & $\begin{aligned} & \mathrm{F} \\
&- \mathrm{F} \\
&= \mathrm{F} \\
&- \mathrm{C} \\
&+ \mathrm{C} \\
&\end{aligned}$ & $\begin{array}{l}F= \\
F-\end{array}$ \\
\hline $\begin{array}{l}115-709 \mathrm{~A}-7 \mathrm{H}-6,65-70 \\
115-709 \mathrm{~A}-7 \mathrm{H}-\mathrm{CC} \\
115-709 \mathrm{~A}-8 \mathrm{H}-4,65-70 \\
115-709 \mathrm{~A}-8 \mathrm{H}-\mathrm{CC} \\
115-709 \mathrm{~A}-9 \mathrm{H}-4,65-70 \\
115-709 \mathrm{~A}-9 \mathrm{H}-\mathrm{CC} \\
115-709 \mathrm{~A}-10 \mathrm{H}-2,65-70\end{array}$ & Stichocorys peregrina & \begin{tabular}{|l|}
$\mathrm{C}$ \\
$\mathrm{C}$ \\
$\mathrm{C}$ \\
$\mathrm{C}$ \\
$\mathrm{C}$ \\
$\mathrm{C}$ \\
$\mathrm{C}$ \\
\end{tabular} & $\begin{array}{l}\mathrm{G} \\
\mathrm{G} \\
\mathrm{G} \\
\mathrm{G} \\
\mathrm{G} \\
\mathrm{G} \\
\mathrm{G} \\
\end{array}$ & $\begin{array}{l}-\bar{R} \\
\mathrm{R} \\
\mathrm{R} \\
\mathrm{R} \\
\mathrm{R} \\
+ \\
\end{array}$ & $\begin{array}{l}R \\
R \\
F\end{array}$ & $\begin{array}{l}\mathrm{R} \\
\mathrm{R} \\
\mathrm{C} \\
\mathrm{F} \\
\mathrm{R} \\
\mathrm{F}\end{array}$ & $\begin{array}{l}- \\
-\end{array}$ & & $\mathrm{F} \begin{array}{l}\mathrm{F} \\
\mathrm{F} \\
\mathrm{F} \\
\mathrm{F}\end{array}$ & & & 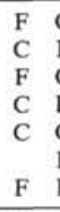 & $\begin{array}{ll}\mathrm{C} & \mathrm{F} \\
\mathrm{F} & \mathrm{C} \\
\mathrm{C} & \mathrm{F} \\
\mathrm{R} & \mathrm{R} \\
\mathrm{C} & \mathrm{C} \\
\mathrm{F} & \mathrm{F} \\
\mathrm{F} & \mathrm{F} \\
\end{array}$ & $\begin{array}{l}\mathrm{F} \\
\mathrm{F} \\
\mathrm{R} \\
\mathrm{R} \\
\mathrm{R} \\
\mathrm{R}\end{array}$ & & $\begin{array}{l}\mathrm{R} \\
\mathrm{R} \\
\mathrm{R} \\
\mathrm{R} \\
\mathrm{R} \\
\mathrm{F} \\
\mathrm{R}\end{array}$ & $\begin{array}{l}\mathrm{R} \\
\mathrm{R} \\
\mathrm{R} \\
\mathrm{R} \\
\mathrm{F} \\
\mathrm{F} \\
\end{array}$ & $\mathrm{R}$ & $\begin{array}{ll}+ & \mathrm{C} \\
\mathrm{R} & \mathrm{C} \\
\mathrm{R} & \mathrm{C} \\
\mathrm{R} & \mathrm{C} \\
\mathrm{R} & \mathrm{C} \\
\mathrm{R} & \mathrm{C} \\
\mathrm{F} & \mathrm{C} \\
\end{array}$ & $\mathrm{F}$ \\
\hline $\begin{array}{l}115-709 \mathrm{~A}-10 \mathrm{H}-4,65-70 \\
115-709 \mathrm{~A}-10 \mathrm{H}-\mathrm{CC} \\
115-709 \mathrm{~A}-11 \mathrm{H}-4,65-70 \\
115-709 \mathrm{~A}-11 \mathrm{H}-\mathrm{CC} \\
115-709 \mathrm{~A}-12 \mathrm{H}-2,65-70\end{array}$ & Didymocyrtis penultima & $\begin{array}{l}\mathrm{C} \\
\mathrm{C} \\
\mathrm{F} \\
\mathrm{C} \\
\mathrm{R}\end{array}$ & $\begin{array}{l}M \\
G \\
M \\
M \\
M\end{array}$ & $\begin{array}{l}+ \\
\mathrm{R} \\
+ \\
+ \\
-\end{array}$ & & & $\begin{array}{l}\mathrm{R} \\
\mathrm{F} \\
\mathrm{R} \\
\mathrm{R} \\
\mathrm{R}\end{array}$ & $\begin{array}{l}\overline{-} \\
\overline{\mathrm{R}}= \\
\overline{+}-\end{array}$ & $\begin{array}{l}\mathrm{F} \\
\mathrm{C} \\
\mathrm{R} \\
\mathrm{R} \\
\mathrm{R}\end{array}$ & & & $\begin{array}{ll}\mathrm{F} & \\
\mathrm{R} & \\
\mathrm{R} & \end{array}$ & $\begin{array}{ll}\mathrm{F} & \mathrm{R} \\
\mathrm{F} & \\
\mathrm{F} & \\
\mathrm{R} & \end{array}$ & R & & $\mathbf{F}$ & $\begin{array}{l}\mathrm{R} \\
- \\
-\end{array}$ & & $\begin{array}{ll}\mathrm{F} & \mathrm{R} \\
\mathrm{C} & \mathrm{R} \\
\mathrm{F} & \mathrm{R} \\
\mathrm{F} & \mathrm{R} \\
\mathrm{F} & \mathrm{R}\end{array}$ & \\
\hline
\end{tabular}


Table 4. Radiolarians from Hole 709C.

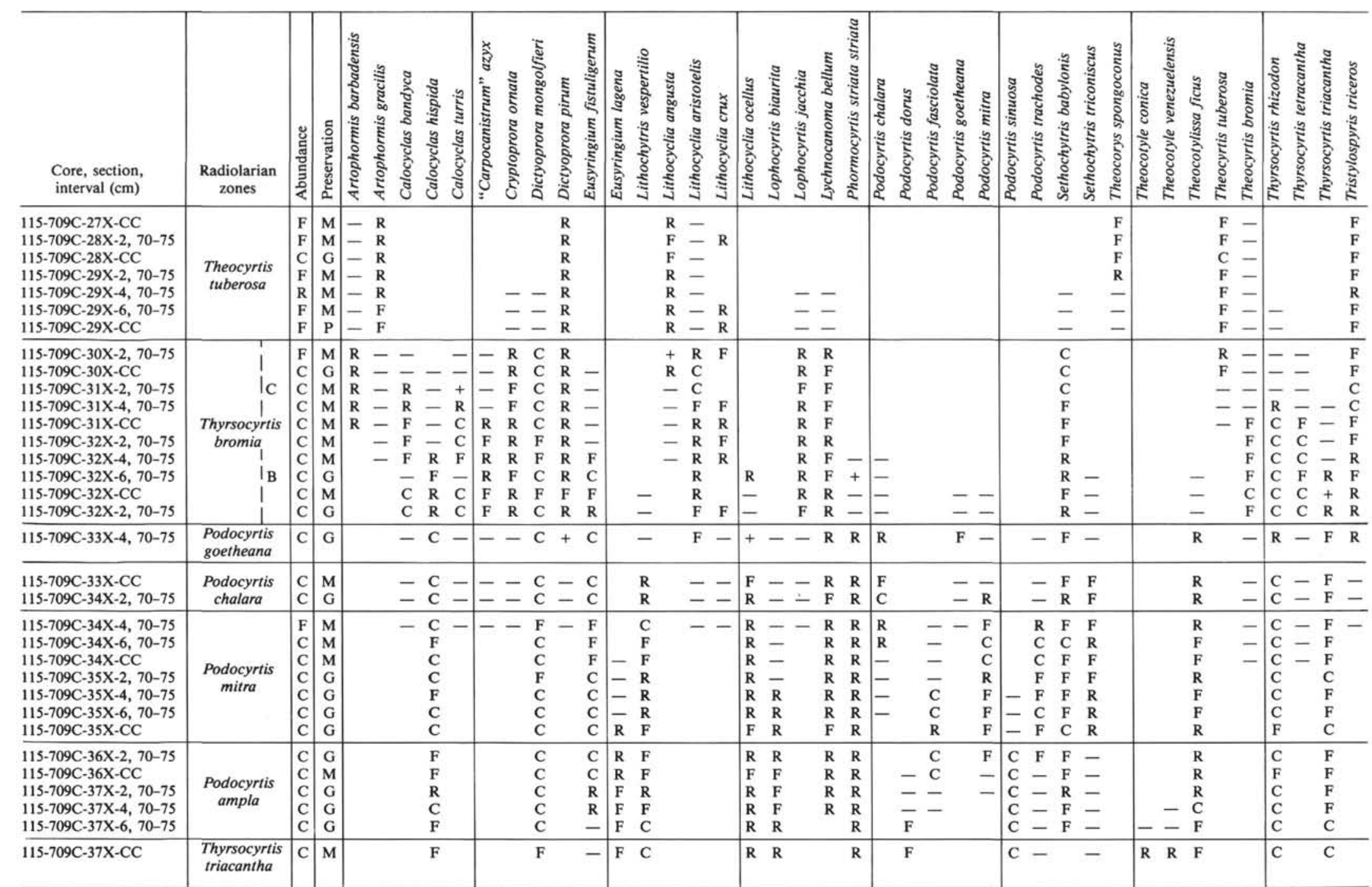


Table 5. Radiolarians from Hole 710A.

\begin{tabular}{|c|c|c|c|c|c|c|c|c|c|c|c|c|c|c|c|c|c|c|c|c|c|c|c|}
\hline $\begin{array}{l}\text { Core, section, } \\
\text { interval }(\mathrm{cm})\end{array}$ & $\begin{array}{l}\text { Radiolarian } \\
\text { zones }\end{array}$ & & 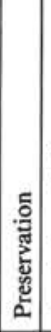 & 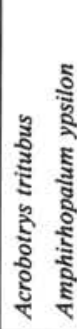 & 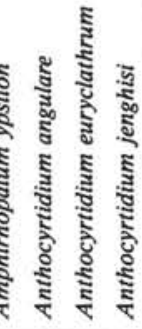 & 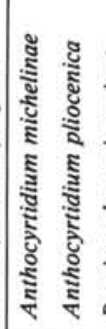 & 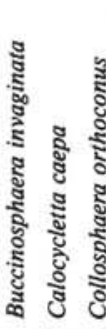 & 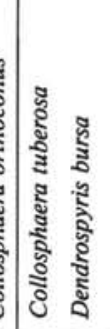 & 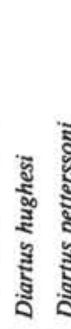 & 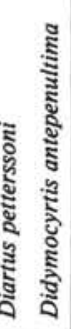 & 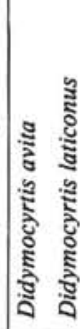 & 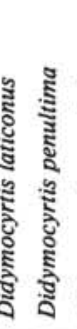 & 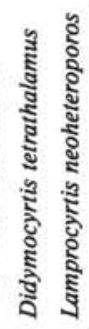 & 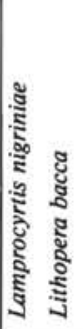 & 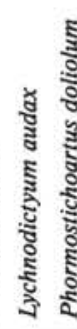 & 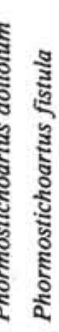 & 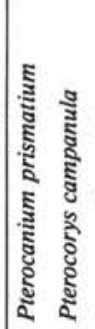 & 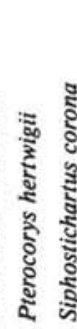 & 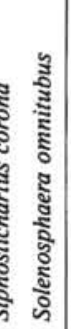 & 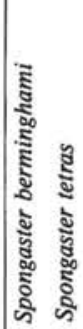 & 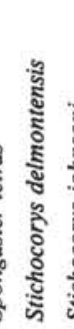 & 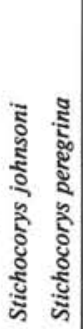 & 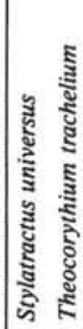 \\
\hline $115-710 \mathrm{~A}-1 \mathrm{H}-2,70-75$ & $\begin{array}{c}\text { Collosphaera } \\
\text { tuberosa }\end{array}$ & C & $\mathrm{G}$ & $\mathbf{F}$ & $F-F-$ & - & - & $\mathbf{R}$ & & & & & F - & $\mathrm{R} \quad \mathrm{R}$ & & & $-F$ & $\mathrm{~F}$ & & $\mathrm{~F}$ & & & $-F$ \\
\hline $\begin{array}{l}115-710 \mathrm{~A}-1 \mathrm{H}-4,70-75 \\
115-710 \mathrm{~A}-1 \mathrm{H}-\mathrm{CC}\end{array}$ & $\begin{array}{l}\text { Amphirhopalum } \\
\text { ypsilon }\end{array}$ & $\begin{array}{l}\mathrm{C} \\
\mathrm{C}\end{array}$ & \begin{tabular}{l|}
$\mathrm{G}$ \\
$\mathrm{M}$
\end{tabular} & $\begin{array}{l}\text { C } \\
\text { F }\end{array}$ & $\begin{array}{l}C=R- \\
F-R-\end{array}$ & - & $\overline{-}$ & - & & & & & $\begin{array}{l}\mathrm{C}- \\
\mathrm{F}-\end{array}$ & $\begin{array}{|ll|}R & R \\
F & R \\
\end{array}$ & & & $\begin{array}{l}-\mathrm{F} \\
-\mathrm{F}\end{array}$ & $\underline{\mathrm{C}}$ & & $\begin{array}{l}\mathbf{R} \\
\mathbf{R}\end{array}$ & & & $\begin{array}{|ll|}\mathbf{R} & \mathrm{C} \\
\mathrm{R} & \mathrm{R} \\
\end{array}$ \\
\hline $\begin{array}{l}115-710 \mathrm{~A}-2 \mathrm{H}-2,70-75 \\
115-710 \mathrm{~A}-2 \mathrm{H}-4,70-75\end{array}$ & $\begin{array}{c}\text { Anthocyrtidium } \\
\text { angulare }\end{array}$ & $\begin{array}{l}\mathrm{C} \\
\mathrm{C}\end{array}$ & \begin{tabular}{|l|}
$G$ \\
$G$
\end{tabular} & $\begin{array}{l}\mathrm{C} \\
\mathrm{F}\end{array}$ & $\begin{array}{l}\mathrm{R}-- \\
\mathrm{R}--\end{array}$ & $\bar{R}$ & - & - & & & & & $\begin{array}{ll}\mathrm{C} & \mathrm{F} \\
\mathrm{C} & \mathrm{F}\end{array}$ & - & & & $\begin{array}{l}-F \\
-\end{array}$ & $\overline{-}$ & & & & & \begin{tabular}{|ll}
$\mathrm{F}$ & $\mathrm{C}$ \\
$\mathrm{R}$ & $\mathrm{R}$ \\
\end{tabular} \\
\hline $\begin{array}{l}115-710 \mathrm{~A}-2 \mathrm{H}-6,70-75 \\
115-710 \mathrm{~A}-2 \mathrm{H}-\mathrm{CC} \\
115-710 \mathrm{~A}-3 \mathrm{H}-2,70-75\end{array}$ & $\begin{array}{l}\text { Pterocanium } \\
\text { prismatium }\end{array}$ & $\begin{array}{l}\mathrm{F} \\
\mathrm{C} \\
\mathrm{F}\end{array}$ & \begin{tabular}{|l|}
$\mathrm{M}$ \\
$\mathrm{G}$ \\
$\mathrm{G}$
\end{tabular} & $\begin{array}{l}\mathrm{F} \\
\mathrm{F} \\
\mathrm{F}\end{array}$ & $\begin{array}{l}-- \\
\bar{F} \\
\bar{F}\end{array}$ & $\begin{array}{l}\mathrm{R} \\
\mathrm{F} \\
\mathrm{R}\end{array}$ & & & & & - & & $\begin{array}{ll}\mathrm{C} & \mathrm{F} \\
\mathrm{R} & \mathrm{F} \\
\mathrm{R} & \mathrm{R}\end{array}$ & $\overline{-}_{-}$ & $=$ & -1 & $\begin{array}{ll}\mathrm{F} & \mathrm{F} \\
\mathrm{R} & \mathrm{F} \\
\mathrm{R} & \mathrm{R}\end{array}$ & & & $\mathbf{R}$ & & $\begin{array}{l}- \\
- \\
-\end{array}$ & $\begin{array}{|ll|}R & R \\
R & F\end{array}$ \\
\hline $\begin{array}{l}115-710 \mathrm{~A}-3 \mathrm{H}-3,95-97 \\
115-710 \mathrm{~A}-3 \mathrm{H}-\mathrm{CC} \\
115-710 \mathrm{~A}-4 \mathrm{H}-2,70-75 \\
115-710 \mathrm{~A}-4 \mathrm{H}-4,70-75 \\
115-710 \mathrm{~A}-4 \mathrm{H}-6,70-75\end{array}$ & $\begin{array}{c}\text { Spongaster } \\
\text { pentas }\end{array}$ & $\begin{array}{l}\mathrm{F} \\
\mathrm{C} \\
\mathrm{C} \\
\mathrm{C} \\
\mathrm{C}\end{array}$ & \begin{tabular}{|l|}
$G$ \\
$G$ \\
$G$ \\
$G$ \\
$G$
\end{tabular} & $\begin{array}{r}\text { F } \\
R \\
+ \\
-- \\
--\end{array}$ & $\begin{array}{l}\mathrm{F} \\
\mathrm{F} \\
\mathrm{F} \\
\mathrm{R} \\
\mathrm{F}\end{array}$ & $\begin{array}{ll}\mathrm{F} & - \\
\mathrm{F} & \mathrm{R} \\
\mathrm{C} & \mathrm{R} \\
\mathrm{C} & \mathrm{F} \\
\mathrm{F} & \mathrm{R}\end{array}$ & & & & & $\begin{array}{l}\bar{R} \\
\text { R } \\
\text { F } \\
\text { F }\end{array}$ & - & $\begin{array}{ll}\mathrm{F} & \mathrm{R} \\
\mathrm{F} & \\
\mathrm{F} & \\
\mathrm{R} & \\
\mathrm{R} & \end{array}$ & C & $\begin{array}{ll}- & - \\
\bar{R} & \\
\mathrm{~F} \\
\mathrm{R} & \mathrm{F} \\
\mathrm{F} & \mathrm{C}\end{array}$ & $\begin{array}{l}- \\
+ \\
R \\
R \\
R \\
R\end{array}$ & \begin{tabular}{|ll}
$\mathrm{F}$ & $\mathrm{R}$ \\
$\mathrm{F}$ & $\mathrm{F}$ \\
$\mathrm{C}$ & \\
$\mathrm{R}$ & \\
$\mathrm{F}$ & \\
\end{tabular} & Z & -- & $\begin{array}{r}\quad F \\
\quad F \\
-\quad \\
-\end{array}$ & $\begin{array}{l}\overline{-} \\
\overline{-} \\
=\end{array}$ & $\begin{array}{r}\mathrm{F} \\
\mathrm{C} \\
\mathrm{C} \\
-\mathrm{C} \\
-\mathrm{C} \\
\end{array}$ & \\
\hline $\begin{array}{l}115-710 \mathrm{~A}-4 \mathrm{H}-\mathrm{CC} \\
115-710 \mathrm{~A}-6 \mathrm{H}-2,75-80 \\
115-710 \mathrm{~A}-6 \mathrm{H}-4,40-50 \\
115-710 \mathrm{~A}-6 \mathrm{H}-6,75-80 \\
115-710 \mathrm{~A}-6 \mathrm{H}-\mathrm{CC} \\
115-710 \mathrm{~A}-7 \mathrm{H}-2,70-75\end{array}$ & $\begin{array}{l}\text { Stichocorys } \\
\text { peregrina }\end{array}$ & $\begin{array}{l}\mathrm{C} \\
\mathrm{C} \\
\mathrm{C} \\
\mathrm{C} \\
\mathrm{F} \\
\mathrm{C} \\
\end{array}$ & \begin{tabular}{|l|}
$\mathrm{G}$ \\
$\mathrm{G}$ \\
$\mathrm{G}$ \\
$\mathrm{G}$ \\
$\mathrm{M}$ \\
$\mathrm{G}$ \\
\end{tabular} & $\begin{array}{l}\bar{R}- \\
\mathrm{R} \\
\mathrm{R} \\
\mathrm{R} \\
\mathrm{R}\end{array}$ & $R$ & $\begin{array}{ll}\mathrm{F} & \mathrm{R} \\
\mathrm{F} & \mathrm{F} \\
& \mathrm{R} \\
\mathrm{R} & \mathrm{F} \\
& \mathrm{F}\end{array}$ & & $\frac{-}{\mathrm{R}}$ & - & $\begin{array}{l}- \\
-\end{array}$ & $\begin{array}{l}\text { F } \\
\text { C } \\
\text { F } \\
\text { C } \\
\text { F } \\
\text { R }\end{array}$ & $\begin{array}{l}- \\
\bar{R} \\
F \\
F \\
R\end{array}$ & & C & $\begin{array}{ll}\mathrm{R} & \mathrm{C} \\
\mathrm{F} & \mathrm{C} \\
\mathrm{R} & \mathrm{C} \\
\mathrm{F} & \mathrm{C} \\
\mathrm{F} & \mathrm{R} \\
\mathrm{F} & \mathrm{C} \\
\end{array}$ & $\begin{array}{ll} & F \\
C & R \\
C & R \\
C & R \\
R & R \\
C & R\end{array}$ & $\begin{array}{l}+ \\
- \\
- \\
-\end{array}$ & $\begin{array}{l} \\
\mathrm{F} \\
\mathrm{R} \\
\mathrm{R} \\
\mathrm{R} \\
\mathrm{R}\end{array}$ & $\begin{array}{ll} & R \\
R \\
R \\
R \\
R \\
R \\
R \\
\end{array}$ & $\begin{array}{l}\mathrm{R} \\
\mathrm{R} \\
\mathrm{R} \\
\mathrm{R}\end{array}$ & $\begin{array}{ll}+ & - \\
+ & \\
+ & \\
+ & F \\
F & F \\
C & F \\
F & F\end{array}$ & \begin{tabular}{ll|}
$\bar{R}$ & $C$ \\
$R$ & $C$ \\
$R$ & $C$ \\
$F$ & $C$ \\
$R$ & $C$ \\
$R$ & $C$ \\
\end{tabular} & \\
\hline $\begin{array}{l}115-710 \mathrm{~A}-7 \mathrm{H}-4,70-75 \\
115-710 \mathrm{~A}-7 \mathrm{H}-6,70-75 \\
115-710 \mathrm{~A}-7 \mathrm{H}-\mathrm{CC}\end{array}$ & $\begin{array}{c}\text { Didymocyrtis } \\
\text { penultina }\end{array}$ & $\begin{array}{l}\mathrm{C} \\
\mathrm{C} \\
\mathrm{F} \\
\end{array}$ & \begin{tabular}{|l|}
$\mathrm{G}$ \\
$\mathrm{M}$ \\
$\mathrm{M}$ \\
\end{tabular} & $\begin{array}{l}\mathrm{R} \\
\mathrm{R} \\
\\
\end{array}$ & & $\begin{array}{|ll|}R & \\
& R \\
& R \\
& \end{array}$ & + & $\begin{array}{l}\mathrm{R} \\
\mathrm{R}\end{array}$ & $\begin{array}{l}\bar{R} \\
+ \\
\end{array}$ & $\begin{array}{l}\mathrm{R} \\
+ \\
\mathrm{R} \\
\end{array}$ & + & $\begin{array}{l}\text { F } \\
\text { C } \\
\text { F }\end{array}$ & & $\begin{array}{l}\mathrm{F} \\
\mathrm{R}\end{array}$ & $\begin{array}{ll} & \mathrm{C} \\
\mathrm{F} & \mathrm{C} \\
\mathrm{R} & \mathrm{F} \\
\end{array}$ & $\begin{array}{ll} & R \\
C & R \\
C & R\end{array}$ & & & $\begin{array}{l}5 \\
\end{array}$ & & $\begin{array}{ll}\mathrm{C} & \mathrm{F} \\
\mathrm{C} & \mathrm{F} \\
\mathrm{F} & \end{array}$ & \begin{tabular}{ll|}
$R$ & $F$ \\
$R$ & $F$ \\
& $R$
\end{tabular} & \\
\hline $\begin{array}{l}115-710 \mathrm{~A}-8 \mathrm{H}-2,70-75 \\
115-710 \mathrm{~A}-8 \mathrm{H}-4,70-75 \\
115-710 \mathrm{~A}-8 \mathrm{H}-6,70-75 \\
115-710 \mathrm{~A}-8 \mathrm{H}-\mathrm{CC} \\
\end{array}$ & $\begin{array}{l}\text { Didymocyrtis } \\
\text { antepenultima }\end{array}$ & $\begin{array}{l}\mathrm{C} \\
\mathrm{F} \\
\mathrm{F} \\
\mathrm{C} \\
\end{array}$ & \begin{tabular}{|l|}
$\mathrm{M}$ \\
$\mathrm{M}$ \\
$\mathrm{P}$ \\
$\mathrm{G}$ \\
\end{tabular} & - & & $\mathbf{R}$ & $\overline{-}$ & $\mathrm{F}$ & $\begin{array}{l}\mathrm{C} \\
\mathrm{R} \\
\mathrm{R} \\
\mathrm{F}\end{array}$ & $\begin{aligned} &- C \\
&- R \\
&- R \\
&- C \\
&\end{aligned}$ & $\begin{array}{l}R \\
+ \\
+\end{array}$ & $\begin{array}{r} \\
R \\
R \\
+ \\
+ \\
+\end{array}$ & & $\begin{array}{l}\mathbf{R} \\
\mathbf{R}\end{array}$ & $\begin{array}{ll}\mathrm{R} & \mathrm{R} \\
\mathrm{F} & \\
\mathrm{F} & \\
\mathrm{F} & \mathrm{F} \\
\end{array}$ & $\begin{array}{l}R \\
R\end{array}$ & & & & & $\begin{array}{l}\mathrm{R} \\
\mathrm{R} \\
\mathrm{R} \\
\mathrm{F} \\
\end{array}$ & $\begin{array}{l}- \\
- \\
-\end{array}$ & \\
\hline $\begin{array}{l}115-710 \mathrm{~A}-9 \mathrm{H}-2,70-75 \\
115-710 \mathrm{~A}-9 \mathrm{H}-4,70-75 \\
115-710 \mathrm{~A}-9 \mathrm{H}-6,70-75\end{array}$ & $\begin{array}{c}\text { Diartus } \\
\text { petterssoni }\end{array}$ & $\begin{array}{l}\mathrm{F} \\
\mathrm{F} \\
\mathrm{F}\end{array}$ & \begin{tabular}{|l|}
$\mathrm{M}$ \\
$\mathrm{P}$ \\
$\mathrm{P}$
\end{tabular} & & & & $\frac{\overline{-}}{\mathrm{R}}$ & & $\begin{array}{l}+\mathrm{F} \\
-\mathrm{R} \\
-\mathrm{R}\end{array}$ & $\begin{array}{l}\mathrm{F}- \\
\mathrm{R}= \\
\mathrm{R}-\end{array}$ & $\begin{array}{l}\mathrm{R} \\
\mathrm{R} \\
\mathrm{R}\end{array}$ & $\begin{array}{l}\mathbf{R}- \\
\mathbf{R} \\
\mathbf{R}\end{array}$ & & & $\begin{array}{ll}\mathrm{F} & \mathrm{R} \\
\mathrm{F} & \end{array}$ & $\begin{array}{l}R \\
R\end{array}$ & & $\mathrm{R}$ & 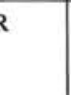 & & $\begin{array}{l}\mathrm{F} \\
\mathrm{F} \\
\mathrm{F}\end{array}$ & $\begin{array}{l}- \\
-\end{array}$ & \\
\hline
\end{tabular}


Table 6. Radiolarians from Hole 711A.

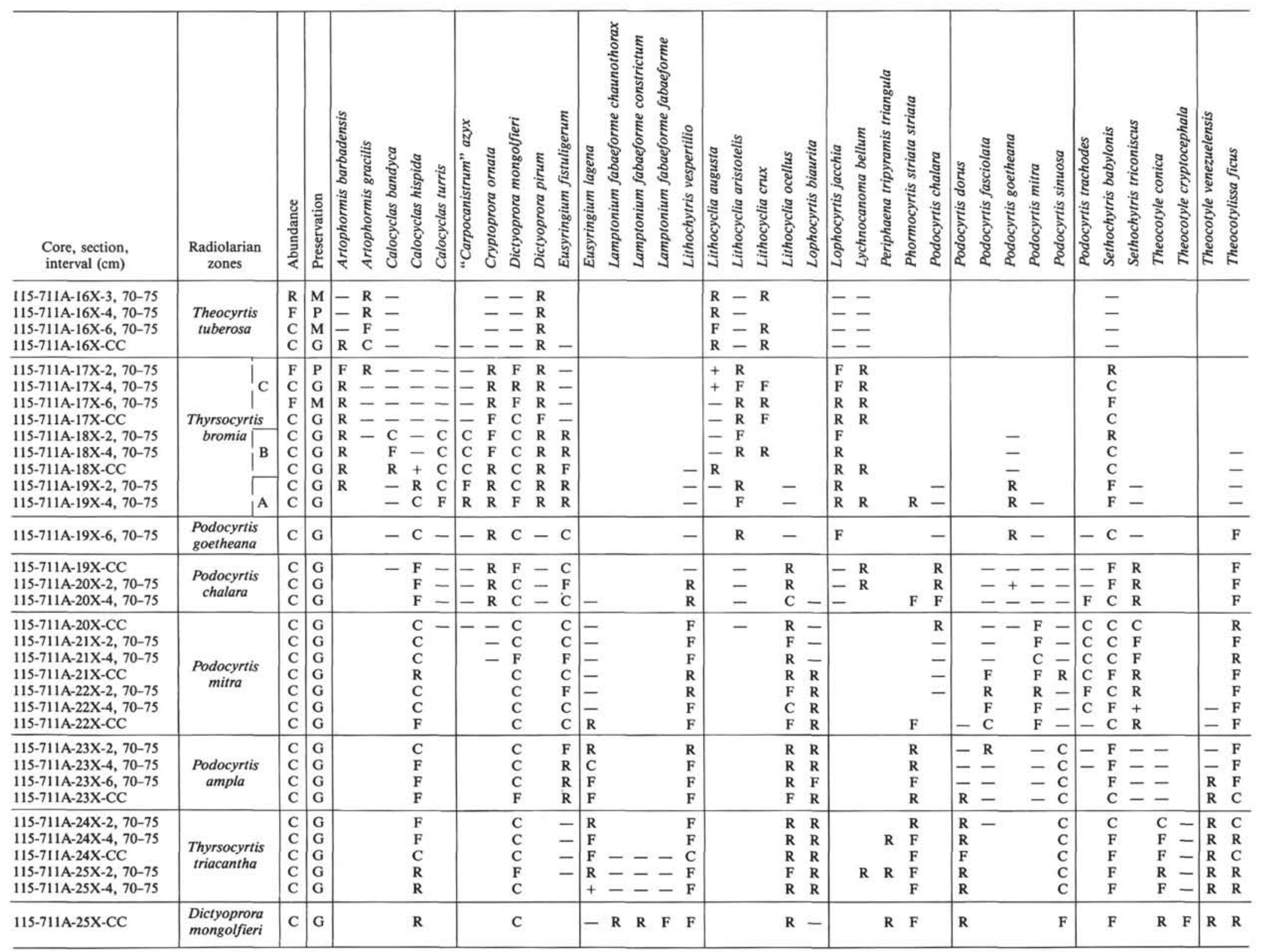


Table 7. Coring intervals and age ranges for the sites in which radiolarians were recovered.

\begin{tabular}{ccl}
\hline Hole & Depth (mbsf) & \multicolumn{1}{c}{ Age range } \\
\hline 707A & $0-213.3$ & Holocene-late Eocene \\
707B & $0-124.9$ & $\begin{array}{l}\text { Holocene-middle Miocene } \\
\text { early Oligocene-Paleocene }\end{array}$ \\
707C & $183.8-443.2$ & Holocene-earliest Miocene \\
709A & $0-203.1$ & Holocene-early Oligocene \\
709B & $0-254.8$ & Holocene-middle Eocene \\
709C & $0-353.7$ & Holocene-early Oligocene \\
710A & $0-209.7$ & Holocene-late Miocene \\
710B & $0-83.4$ & Holoce \\
711A & $0-249.7$ & Holocene-middle Eocene \\
711B & $0-98.6$ & Holocene-early Oligocene \\
712A & $0-115.3$ & Holocene-middle Miocene \\
713A & $0-191.7$ & Holocene-middle Eocene \\
714A & $0-233.0$ & Holocene-late Oligocene \\
714B & $0-122.6$ & Holocene-middle Miocene \\
\hline
\end{tabular}

nis, and Calocyclas turris. The absence of Thyrsocyrtis bromia and $T$. tetracantha is consistent with this zonal determination.

Samples 115-707A-23X-6, $101 \mathrm{~cm}$, through 115-707A-23XCC (211.2-212.3 mbsf) contain well-preserved radiolarians diagnostic of the Calocyclas bandyca Zone of late Eocene age. Key taxa include $T$. bromia, $T$. tetracantha, C. turris, C. ornata, C. azyx, T. rhizodon, C. bandyca, E. fistuligerum, D. mongolfieri, D. pirum, T. triceros, and L. jacchia.

\section{Hole 707 C}

Hole $707 \mathrm{C}$ contains identifiable radiolarians only in Cores 115-707C-3R through -11R (193.5-280.3 mbsf). The Theocyrtis tuberosa Zone of early Oligocene age is well represented in Core 115-707C-3R (193.5-203.1 mbsf). Diagnostic taxa in this interval include T. tuberosa, Lithocyclia angusta, and Artophormis gracilis. Core 115-707C-4R (203.1-212.8 mbsf) obtained zero core recovery, and thus the Eocene/Oligocene boundary interval was not recovered in this hole.

Samples 115-707C-5R-CC through 115-707C-6R-CC (222.5$232.1 \mathrm{mbsf}$ ) may be assigned to the Calocyclas bandyca Zone of late Eocene age. Diagnostic taxa include T. bromia, T. tetracantha, C. turris, D. pirum, D. mongolfieri, C. azyx, C. bandyca, L. bellum, L. aristotelis, T. rhizodon, S. babylonis, and L. jacchia.

A short unconformity is apparently present in the interval between Samples 115-707C-6R-CC and 115-707C-7R-2, $78 \mathrm{~cm}$ (232.1-234.4 mbsf). Samples 115-707C-7R-2, $78 \mathrm{~cm}$, to $115-$ 707C-11R-CC (234.4-280.3 mbsf) may be assigned to the Podocyrtis mitra Zone of middle Eocene age. Diagnostic taxa include Podocyrtis mitra, P. trachodes, Sethochytris triconiscus, Calocyclas hispida, Lithocyclia ocellus, Podocyrtis helenae, Lithochytris vespertilio, and Thyrsocyrtis triacantha.

All samples below Core 115-707C-11R (280.3 mbsf) are barren of radiolarians.

\section{Site 708}

A single hole (708A) was drilled at Site 708, located in the basin separating the Madingley Rise from the Mascarene Plateau, at a water depth of $4109 \mathrm{~m}$. The entire cored interval contains graded calcareous turbidites, ranging in thickness from a few millimeters up to several tens of centimeters. Within the entire interval cored (0-236.2 mbsf) the cumulative thickness of the turbidites is comparable to, and perhaps greater than, that of the intervening pelagic layers. Because of the predominance of turbidites, and the doubtful reliability of detailed stratigraphic studies at this site, only core-catcher samples were examined.
Radiolarians are common and well preserved only in the upper 13 cores at Site 708 (0-120.6 mbsf). Radiolarian fragments in Cores 115-708A-14X through $-17 \mathrm{X}$ (130.3-159.3 mbsf) are sparse and nondiagnostic. Radiolarians in Cores $115-708 \mathrm{~A}-18 \mathrm{X}$ through $-25 \mathrm{X}$ (168.6-236.2 mbsf) are rare and poorly preserved, and allow only an approximate zonal age determination.

From a preliminary examination of the upper 13 cores, we were able to make the following zonal assignments:

Samples 115-708A-2H-CC through 115-708A-3H-CC (18.6$28.1 \mathrm{mbsf}$ ) are of Quaternary age; diagnostic taxa include $T$. trachelium, A. ypsilon, D. tetrathalamus, L. nigriniae, S. universus, and $L$. bacca.

Sample $115-708 \mathrm{~A}-4 \mathrm{H}-\mathrm{CC}$ ( $37.6 \mathrm{mbsf}$ ) belongs to the $P$. prismatium Zone of late Pliocene age. Diagnostic taxa include $A$. ypsilon, $P$. prismatium, T. trachelium, and $T$. vetulum.

Samples 115-708A-5H-CC through 115-708A-7H-CC may be assigned to the $S$. pentas Zone of early Pliocene age. Diagnostic taxa include $A$. jenghisi, $P$. prismatium, $S$. peregrina, $A$. michelinae, L. audax, and $P$. fistula.

Samples 115-708A-8H-CC through 115-708A-12X-CC (76.0$111.0 \mathrm{mbsf}$ ) may be assigned to the Stichocorys peregrina Zone of late Miocene age. Diagnostic taxa in these samples include $S$. omnitubus, $P$. doliolum, $S$. peregrina, A. jenghisi, D. penultima, $P$. fistula, A. michelinae, $L$. audax, $S$. johnsoni, and $L$. bacca.

Sample 115-708A-13X-CC may be assigned to the $D$. antepenultima Zone of late Miocene age. Diagnostic taxa include $D$. antepenultima, L. audax, S. delmontensis, and D. hughesi.

Samples 115-708A-14X-CC through 115-708A-17X-CC (130.3$159.3 \mathrm{mbsf}$ ) are barren of diagnostic radiolarians.

Sample $115-708 \mathrm{~A}-18 \mathrm{X}-\mathrm{CC}$ (168.6 mbsf) is tentatively assigned to the Cyrtocapsella tetrapera Zone of early Miocene age. Indicative taxa include $C$. virginis, $C$. serrata, and $D$. prismatica.

Sample 115-708A-19X-CC (178.2 mbsf) is assigned to the $L$. elongata Zone of earliest Miocene age. Taxa include L. elongata and $C$. robusta.

Samples 115-708A-20X-CC through 115-708A-23X-CC (187.9216.8 mbsf) may be assigned to the Dorcadospyris ateuchus Zone of late Oligocene age. Diagnostic taxa include $D$, ateuchus, D. papilio, D. prismatica, and C. pegetrum.

Samples 115-708A-24X-CC through 115-708A-25X-CC (226.5$236.2 \mathrm{mbsf}$ ) may be assigned to the Theocyrtis tuberosa Zone of early Oligocene age. Diagnostic taxa include $L$. angusta, $D$. pirum, A. gracilis, and T. spongoconus.

\section{Site 709}

Three holes, all within $0.1 \mathrm{~km}$ of each other, were drilled at Site 709, located on the summit of the Madingley Rise, in a water depth of $3041 \mathrm{~m}$. Table 7 lists the intervals cored, all of which had excellent to good core recovery. Radiolarians were examined in selected intervals from each of the three holes in order to form a composite stratigraphic section. The following intervals were sampled: (1) Hole 709A, 0-203.1 mbsf; (2) Hole 709B, 189.4-254.8 mbsf; and (3) Hole 709C, 256.7-353.7 mbsf.

In the discussion that follows, the composite stratigraphic section at this site will be summarized from top to bottom.

\section{Late Miocene to Holocene}

Radiolarian assemblages are common and well preserved in this interval, extending between Sample 115-709A-1H-2, $75 \mathrm{~cm}$, and Sample $115-709 \mathrm{~A}-12 \mathrm{H}-2,65 \mathrm{~cm}$ (2.2-108.8 mbsf). Diagnostic taxa and zonal age assignments are presented in Table 3.

\section{Early Miocene to Middle Miocene}

Radiolarians are absent in the interval between Samples 115$709 \mathrm{~A}-12 \mathrm{H}-4,65 \mathrm{~cm}$, and 115-709A-19H-CC (111.8-183.9 mbsf), 
which corresponds to the middle and early Miocene on the basis of calcareous microfossils.

\section{Oligocene}

Radiolarians are rare and moderately to poorly preserved in the Oligocene interval of Holes 709A and 709B. For this reason, the biostratigraphic zonal determinations will be summarized briefly in the text rather than tabulated.

Samples 115-709A-20H-2, $65 \mathrm{~cm}$, through 115-709A-20H-6, $65 \mathrm{~cm}$ (186.1-192.1 mbsf), and Samples 115-709B-21X-2, 65 $\mathrm{cm}$, through 115-709B-22X-2, $65 \mathrm{~cm}$ (189.4-199.0 mbsf), may be assigned to the Lychnocanoma elongata Zone of latest Oligocene and earliest Miocene age. Diagnostic taxa include L. elongata, Dorcadospyris ateuchus, and D. papilio.

Samples 115-709B-22X-4, $65 \mathrm{~cm}$, through 115-709B-25X-CC (202.0-235.5 mbsf) may be assigned to the Dorcadospyris ateuchus Zone of late Oligocene age. Diagnostic taxa include Dorcadospyris ateuchus, D. papilio, Cyclampterium pegetrum, Calocycletta robusta, Artophormis gracilis, and Didymocyrtis prismatica.

Samples 115-709B-26X-2, $65 \mathrm{~cm}$, to 115-709B-27X-CC (237.7$254.8 \mathrm{mbsf}$ ), and Samples $115-709 \mathrm{C}-27 \mathrm{X}-\mathrm{CC}$ to $115-709 \mathrm{C}-29 \mathrm{X}-\mathrm{CC}$ (256.7-276.1 mbsf) may be assigned to the Theocyrtis tuberosa Zone of early Oligocene age. Diagnostic taxa within this interval include Tristylospyris triceros, Theocyrtis tuberosa, Lithocyclia angusta, Artophormis gracilis, Dictyoprora pirum, and Didymocyrtis prismatica.

Radiolarian abundance increases and preservation improves downward within the Oligocene. The assemblages become quite diverse and well preserved as one approaches the Eocene/Oligocene boundary.

\section{Middle and Late Eocene}

Samples 115-709C-30X-2, $70 \mathrm{~cm}$, through 115-709C-37X-CC (278.3-353.7 mbsf) contain common and moderately to well-preserved radiolarians that span the Eocene/Oligocene boundary and continue down to the Thyrsocyrtis triacantha Zone of middle Eocene age. Diagnostic taxa and zonal age assignments for this interval are presented in Table 4.

The radiolarian occurrences in Core 115-709C-33X indicate that there may be a short unconformity between Sections 2 and 4, or between 307.3 and 310.3 mbsf (see Table 4). The nannofossil assemblages from this interval show significant reworking and cannot be used to verify whether or not the unconformity is present (H. Okada, pers. comm., 20 August 1988).

\section{Site 710}

Two holes, located within $0.1 \mathrm{~km}$ of each other, were drilled at Site 710 , located on the flank of the Madingley Rise, in a water depth of $3824 \mathrm{~m}$. The intervals that were cored are given in Table 7, all with excellent to good core recovery.

Because the site was double-cored, only the assemblages in Hole 710A were examined. However, a few samples in the Pliocene of Hole $710 \mathrm{~B}$ were checked to confirm the zonal boundary determinations, as Core $115-710 \mathrm{~B}-4 \mathrm{H}$ of Hole $710 \mathrm{~A}$ had no core recovery.

Hole $710 \mathrm{~A}$ contains common radiolarian assemblages only within the Holocene to late Miocene interval (Cores 115-710A$1 \mathrm{H}$ through $-9 \mathrm{H} ; 0-86.2 \mathrm{mbsf}$ ). Table 5 lists the diagnostic taxa and zonal age assignments for this stratigraphic interval.

Samples 115-710A-10H-2, $70 \mathrm{~cm}$, through 115-710A-14X-4, $70 \mathrm{~cm}$ (88.4-130.1 mbsf) are barren of radiolarians.

Samples 115-710A-14X-CC through 115-710A-15X-CC contain rare and poorly preserved specimens that are tentatively assigned to either the Cyrtocapsella tetrapera or the Lychnocanoma elongata zones of early Miocene age. Taxa in these sam- ples include Calocycletta robusta, Dorcadospyris ateuchus, and Dendrospyris bursa.

Samples 115-710A-16X-4, $70 \mathrm{~cm}$, through 115-710A-20X-4, $70 \mathrm{~cm}$ (147.3-186.0 mbsf), may be assigned to the Dorcadospyris ateuchus Zone of late Oligocene age. Diagnostic taxa include Dorcadospyris ateuchus, D. papilio, Theocorys spongoconus, Lithocyclia angusta, Theocyrtis annosa, Didymocyrtis prismatica, and Cyclampterium milowi.

Samples 115-710A-20X-CC through 115-710A-22X-CC (190.4$209.7 \mathrm{mbsf}$ ) may be assigned to the Theocyrtis tuberosa Zone of early Oligocene age. Taxa present in these samples include Theocyrtis tuberosa, Lithocyclia angusta, Tristylospyris triceros, Dictyoprora pirum, and Lithocyclia crux.

\section{Site 711}

Two holes, located within $0.1 \mathrm{~km}$ of each other, were drilled at Site 711 , located near the base of the Madingley Rise in a water depth of $4430 \mathrm{~m}$. The stratigraphic intervals that were cored are listed in Table 7; all had excellent to good core recovery. Because the interval cored in Hole 711B duplicated that of Hole 711A, only samples from Hole 711A were examined for this study.

Radiolarians are common and well preserved only in two stratigraphic intervals at this site: the Quaternary and the earliest Oligocene through middle Eocene.

\section{Quaternary}

Sample $115-711 \mathrm{~A}-1 \mathrm{H}-2,70 \mathrm{~cm}$, may be assigned to the $B$. invaginata Zone. Diagnostic taxa include $B$. invaginata, Collosphaera tuberosa, and Amphirhopalum ypsilon. The middle Brunhes indicator species Stylatractus universus is absent.

Sample $115-711 \mathrm{~A}-1 \mathrm{H}-4,70 \mathrm{~cm}$, may be assigned to the $A$. ypsilon Zone. Diagnostic taxa include Theocorythium trachelium, Didymocyrtis tetrathalamus, Amphirhopalum ypsilon, Pterocorys hertwigii, and rare specimens of Stylatractus universus. The late Pleistocene forms $B$. invaginata and C. tuberosa and the early Pleistocene form Anthocyrtidium angulare are absent.

Sample 115-711A-1H-CC may be assigned to the Anthocyrtidium angulare Zone. Taxa in this sample include $A$. angulare, A. michelinae, Amphirhopalum ypsilon, Didymocyrtis tetrathalamus, Theocorythium trachelium, and Stylatractus universus.

\section{Pliocene to Early Oligocene}

Only one sample in this stratigraphic interval contained identifiable radiolarians. Sample 115-711A-2H-2, $70 \mathrm{~cm}$, contains rare and moderately preserved taxa indicative of the Pterocanium prismatium Zone. Forms present include $P$. prismatium, $T$. trachelium, and common Eocene taxa that are evidently reworked.

All samples between 115-711A-2H-CC and 115-711A-12X-2, $70 \mathrm{~cm}(17.7-106.7 \mathrm{mbsf})$, are barren of radiolarians.

\section{Early Oligocene}

Samples 115-711A-12X-CC through 115-711A-16X-CC (114.2$153.0 \mathrm{mbsf}$ ) contain radiolarians diagnostic of the Theocyrtis tuberosa Zone of early Oligocene age. Specimens are rare and poorly preserved at the top of this interval and become increasingly abundant and better preserved as one approaches the Eocene/Oligocene boundary. Diagnostic taxa in this interval include $T$. tuberosa, T. triceros, D. pirum, A. gracilis, D. prismatica, and L. angusta.

\section{Late and Middle Eocene}

Samples 115-711A-16X-CC through 115-711A-25X-CC (153.0240.0 mbsf) contain common and well-preserved radiolarian as- 
semblages of late and middle Eocene age. Diagnostic taxa and zonal age assignments are presented in Table 6.

\section{Sites 712 and 713}

Sites 712 and 713 , located approximately $3 \mathrm{~km}$ apart, were drilled on Chagos Bank, in water depths of $2903 \mathrm{~m}$ and $2920 \mathrm{~m}$, respectively. The intervals cored are presented in Table 7, all of which had excellent core recovery.

\section{Hole $712 A$}

In Hole $712 \mathrm{~A}$, identifiable radiolarians are present only in Cores $115-712 \mathrm{~A}-1 \mathrm{R}$ through $-4 \mathrm{R}$ (0-38.3 mbsf).

Sample 115-712A-1R-CC (9.4 mbsf) may be assigned to the $S$. pentas Zone of Pliocene age. Diagnostic taxa include $P$. doliolum, L. audax, S. peregrina, D. avita, and $P$. fistula. Solenosphaera omnitubus is absent.

Sample 115-712A-2R-CC (19.0 mbsf) may be assigned to the $S$. peregrina Zone of earliest Pliocene age. Diagnostic taxa include $P$. doliolum, $S$. peregrina, $S$. omnitubus, L. audax, and D. penultima.

Sample 115-712A-3R-CC (28.6 mbsf) may be assigned to the D. penultima Zone of late Miocene age. Diagnostic taxa include $S$. delmontensis, $P$. doliolum, L. audax, S. omnitubus, D. penultima, and $S$. corona.

Sample 115-712A-4R-CC (38.3 mbsf) may be assigned to the D. antepenultima Zone of late Miocene age. Diagnostic taxa include $D$. antepenultima, $D$. hughesi, S. johnsoni, $P$. fistula, $L$. audax, and $S$. delmontensis.

\section{Hole $713 A$}

In Hole $713 \mathrm{~A}$, identifiable radiolarians of Neogene age are present in Cores $115-713 \mathrm{~A}-1 \mathrm{R}$ through $-4 \mathrm{R}$ (0-30.5 mbsf). Core $115-713 \mathrm{~A}-5 \mathrm{R}$ (32.7-40.1 mbsf) is barren of radiolarians. A significant unconformity lies between Cores $115-713 A-4 R$ and $-5 R$, on the basis of calcareous nannofossils. Cores 115-713A-6R though-11R contain common radiolarians of middle Eocene age. The following age assignments may be made for those stratigraphic intervals containing radiolarians:

Samples 115-713A-1R-CC through 115-713A-2R-4, 70-75 cm (1.6-3.8 mbsf), contain poorly preserved radiolarians of Quaternary age, including $A$. ypsilon, $A$. michelinae, $D$. tetrathalamus, $T$. trachelium, $S$. universus, and $A$. angulare.

Sample 115-713A-2R-CC may be assigned to the $S$. pentas Zone of late Pliocene age. Diagnostic taxa include $P$. doliolum, A. michelinae, L. audax, $S$. peregrina, and $P$. fistula. Solenosphaera omnitubus is absent.

Samples 115-713A-3R-4, $70 \mathrm{~cm}$, through 115-713A-4R-CC (16.4-30.5 mbsf) may be assigned to the $D$. penultima Zone of late Miocene age. Diagnostic taxa include $D$. penultima, $S$. delmontensis, L. audax, S. johnsoni, S. corona, A. michelinae, $P$. doliolum, and $A$. jenghisi.

Samples 115-713A-6R-2, $70 \mathrm{~cm}$, through 115-713A-10R-CC (42.3-88.4 mbsf) may be assigned to the T. triacantha Zone of middle Eocene age. Diagnostic taxa include $T$. triacantha, $E$. fistuligerum, T. rhizodon, E. lagena, C. hispida, D. mongolfieri, S. babylonis, $P$. striata striata, and $L$. ocellus.

Sample 115-713A-11R-3, $70 \mathrm{~cm}$ (92.1 mbsf), may be assigned to the $D$. mongolfieri Zone of middle Eocene age. Diagnostic taxa include $T$. tensa, $P$. sinuosa, L. ocellus, C. hispida, and $D$. mongolfieri.

\section{Site 714}

Two holes were drilled at Site 714 , located on the eastern shoulder of the Maldives Ridge, in a water depth of $2038 \mathrm{~m}$. Table 7 lists the intervals cored, which had excellent core recovery.

Radiolarians were examined only in Hole $714 \mathrm{~A}$, because the sequences obtained in Hole 714B duplicate those in Hole 714A.
The following zonal age assignments may be made on the basis of this examination:

Sample $115-714 \mathrm{~A}-1 \mathrm{H}-\mathrm{CC}(2.8 \mathrm{mbsf})$ may be assigned to the $B$. invaginata Zone of Quaternary age. Diagnostic taxa include B. invaginata, D. tetrathalamus, L. nigriniae, T. trachelium, $A$. ypsilon, and C. tuberosa.

Sample 115-714A-2H-CC (12.4 mbsf) may be assigned to the $C$. tuberosa Zone of Quaternary age. Diagnostic taxa include $C$. tuberosa, C. orthoconus, $S$. tetras, $P$. hertwigii, $A$. ypsilon, and D. tetrathalamus. Stylatractus universus is absent.

Sample 115-714A-3H-4, $70 \mathrm{~cm}$ (17.6 mbsf) may be assigned to the A. ypsilon Zone of Quaternary age. Diagnostic taxa include $S$. universus, D. tetrathalamus, T. trachelium, and $A$. ypsilon.

A significant unconformity occurs between Samples 115 $714 \mathrm{~A}-3 \mathrm{H}-4,70 \mathrm{~cm}$, and 115-714A-3H-CC (17.6-22.0 mbsf).

Samples 115-714A-3H-CC through 115-714A-5H-4, $70 \mathrm{~cm}$ (22.0-36.9 mbsf), may be assigned to the $D$. antepenultima Zone of late Miocene age. Diagnostic taxa present include $D$. antepenultima, $D$. hughesi, $S$. delmontensis, $P$. doliolum, $S$. corona, $L$. audax, C. caepa, D. bursa, and $S$. johnsoni.

Samples 115-714A-5H-CC through 115-714A-10H-4, $70 \mathrm{~cm}$ (41.4-85.0 mbsf), may be assigned to the $D$. petterssoni Zone of late Miocene age. Diagnostic taxa in these samples include $D$. petterssoni, $D$. laticonus, $S$. delmontensis, $C$. caepa, $L$. thornburgi, L. audax, S. corona, L. neotera, P. marylandicus, C. japonica, C. cornuta, and C. cristatum.

Samples 115-714A-10H-CC through 115-714A-14X-4, $70 \mathrm{~cm}$ (89.5-123.6 mbsf), may be assigned to the Dorcadospyris alata Zone of middle Miocene age. Diagnostic taxa include $D$. alata, C. bramlettei, C. caepa, C. cornuta, P. marylandicus, L. audax, $S$. wolffii, $C$. virginis, and C. costata.

Samples 115-714A-14X-CC through 115-714A-17X-CC (126.8$155.7 \mathrm{mbsf}$ ) may be assigned to the Calocycletta costata Zone of middle Miocene age. Diagnostic taxa include $C$. virginis, $S$. wolffii, $C$. costata, $S$. delmontensis, $E$. diaphanes, $P$. marylandicus, C. cornuta, C. bramlettei, and L. elongata.

Samples 115-714A-18X-4, $70 \mathrm{~cm}$, through 115-714A-18X-CC (160.9-165.3 mbsf) may be assigned to the Stichocorys wolffii Zone of early Miocene age. Diagnostic taxa include $C$. cornuta, $S$. wolffii, $C$. virginis, $D$. violina, $S$. delmontensis, and $L$. elongata.

Samples 115-714A-19X-4, $70 \mathrm{~cm}$, through 115-714A-20X-CC (170.5-184.7 mbsf) may be assigned to the Stichocorys delmontensis Zone of early Miocene age. Diagnostic taxa include $S$. delmontensis, C. tetrapera, D. violina, C. caepa, E. cienkowskii, $E$. diaphanes, and $C$. cornuta.

Sample 115-714A-21X-CC (194.4 mbsf) may be assigned to the Cyrtocapsella tetrapera Zone of early Miocene age. Diagnostic taxa include $L$. elongata, D. prismatica, $C$. virginis, and A. gracilis.

Samples 115-714A-22X-4, $70 \mathrm{~cm}$, through 115-714A-23X-CC (199.6-213.8 mbsf) may be assigned to the Lychnocanoma elongata Zone of earliest Miocene or latest Oligocene age. Diagnostic taxa include $A$. gracilis, C. robusta, D. prismatica, C. pegetrum, D. ateuchus, T. annosa, L. trifolium, E. cienkowskii, and D. bursa.

All samples examined below Sample 115-714A-23X-CC (213.8 mbsf) are barren of radiolarians.

\section{DISCUSSION}

\section{Radiolarian Events}

This report documents the succession of radiolarian events in two stratigraphic intervals: 31 events are identified in the late Neogene (0-8 Ma), and 55 events in the middle Eocene to earliest Oligocene (35-49 Ma). Within each of these intervals, there 
are a number of important events that have been identified in drill sites elsewhere, but were not identified in Leg 115 core material because of variations in species abundance and specimen preservation.

In the late Neogene sequences of the central Indian Ocean, Johnson et al. (1989) have identified several datum levels on the basis of the genera Anthocyrtidium and Pterocorys. These two genera were well represented in Leg 115 material, but a number of the taxa occurred only sporadically. Consequently, the following events that were identified previously (Johnson et al., 1989) are omitted from this report:

Top Anthocyrtidium nosicaae
Base Anthocyrtidium euryclathrum
Base Pterocorys zancleus
Top Anthocyrtidium ehrenbergi
Top Anthocyrtidium prolatum

A number of other significant late Neogene events were not possible to identify, including the following:

1. Theocorythium vetulum was virtually absent from the Pliocene sequences; thus, its upper and lower limits were not identified.

2. Specimens of the Spongaster lineage, extending from $S$. berminghami to $S$. pentas to $S$. tetras, were very rare or absent entirely. Thus, they were not satisfactory as a basis for selecting datum levels or defining zonal boundaries. For this reason, the base of the $S$. pentas Zone in the early Pliocene was approximated by the LO of Solenosphaera omnitubus.

3. The evolution of Pterocanium prismatium in the earliest Pliocene occurred over a long period of time (at least $1 \mathrm{~m}$.y.; see Lazarus, 1985). Moreover, specimens of this taxon are very rare in Leg 115 material. The lower limit of this species was not identified therefore.

In the middle and late Eocene interval, the assemblages are remarkably diverse and well preserved in Holes 709C and 711A. However, the Podocyrtis lineage extending from $P$. diamesa to $P$. phyxis to $P$ ampla was totally missing and was not satisfactory, therefore, as a zonal boundary criterion. For this reason, the base of the $P$. ampla Zone was approximated by the LO of Theocotyle conica.

The form Artophormis barbadensis was notably rare in the late Eocene, and its lower limit could not be identified.

\section{Paleomagnetic Calibrations and Diachroneity}

Studies of paleomagnetically dated cores have documented the significance of time-transgressive biostratigraphic events (Johnson and Nigrini, 1985; Barron et al., 1985; Baldauf et al., 1986). One of the objectives of drilling on Leg 115 was to recover a continuous magnetic polarity stratigraphy in the Neogene, and to calibrate microfossil events to the geomagnetic polarity time scale (Berggren et al., 1985). This would allow a more precise assessment of the extent and significance of time-transgressive "events."

Of the Neogene sites drilled on Leg 115, only Site 710 obtained both a reliable paleomagnetic record and radiolarian-rich sediments. The magnetic stratigraphy for this site (Backman et al., in press) allows a precise age-depth curve to be constructed (see Schneider, this volume). Radiolarian events support the polarity designations in at least three significant instances:

1. The LO of Pterocanium prismatium, which is near the top of the Olduvai, is identified between 14.7 and $17.7 \mathrm{mbsf}$ in Hole 710A (see Appendix B). Thus, the normal magnetic event in the lower part of Core 115-710A-2H (Backman et al., in press, fig. 1) appears to be the Olduvai.

2. The LO of Stichocorys peregrina, at about $2.6 \mathrm{Ma}$ in the uppermost Gauss (Johnson et al., 1989), is identified between 21.4 and 23.1 mbsf in Hole 710A (Appendix B). Thus, the normal magnetic event in the top of Core 115-710A-3H (Backman et al., in press, fig. 1) is the uppermost Gauss. The overlying lower Matuyama, around 2.0-2.4 Ma, may be missing, as suggested by Backman et al. (in press).

3. The FO of Amphirhopalum ypsilon, at $3.8 \mathrm{Ma}$ in the upper part of the Gilbert (Johnson et al., 1989), is identified between 31.0 and 34.0 mbsf in Hole 710A (Appendix B). Thus, the long intervals of reversed polarity in Core $115-710 \mathrm{~A}-4 \mathrm{H}$ (Backman et al., in press, fig. 1) appear to correspond to the Gilbert.

The polarity stratigraphy of Hole 710A (Schneider, this volume) can be used to assess absolute ages and the synchroneity of several well-defined radiolarian events:

1. The transition from Stichocorys delmontensis to $S$. peregrina occurs at 59.7-62.7 mbsf (Appendix B). This level corresponds exactly with the FO of the nannofossil $A$. primus, which has been dated in Site 710 as $6.7 \mathrm{Ma}$ (Backman et al., in press, table 1).

2. The FO of Solenosphaera omnitubus also occurs at 59.762.7 mbsf (Appendix A) and may therefore be dated as 6.7 Ma.

3. The LO of Diartus hughesi occurs at 62.7-65.7 mbsf (Appendix B). This level is between the FO of $A$. primus and the FO of D. quinqueramus (6.70 and $7.46 \mathrm{Ma}$, respectively; see Backman et al., in press, table 1) and thus may be dated as approximately $7.0 \mathrm{Ma}$ in Site 710 .

4. The FO of $D$. hughesi occurs at 78.8-81.8 mbsf (Appendix B). This level corresponds with the FO of D. neohamatus, which has been dated as $8.96 \mathrm{Ma}$ in Site 710 (Backman et al., in press, table 1).

If we now compare these age estimates with those derived from paleomagnetically dated core material in the equatorial Pacific (Johnson and Nigrini, 1985), we note that two of the events appear to be synchronous and two are diachronous (see Table 8). From these comparisons of ages, it appears that $D$. hughesi and $S$. peregrina appeared somewhat earlier in the equatorial Indian Ocean than in the equatorial Pacific. This pattern is consistent with earlier observations of Johnson and Nigrini (1985). It is clear that faunal and floral diachroneity remains a significant phenomenon to document and to explain.

\section{Paleoceanography}

In the western tropical Indian Ocean, the deposition of biogenic silica undergoes a dramatic shift at the middle/late Miocene boundary, about $10-11 \mathrm{Ma}$. Below this transition, in sediments of Oligocene through middle Miocene age, silica is ab-

Table 8. Comparison of age estimates for Site 710 (Indian Ocean) and Site 573 (Pacific Ocean).

\begin{tabular}{lccc}
\hline & \multicolumn{2}{c}{ Age (Ma) } \\
\multicolumn{1}{c}{ Event } & Site 710 & Site 573 \\
\hline Stichocorys delmontensis $\rightarrow$ Stichocorys peregrina & 6.7 & $6.1-6.3$ \\
B Solenosphaera omnitubus & 6.7 & $6.7-6.8$ \\
T Diartus hughesi & 7.0 & $7.0-7.1$ \\
B Diartus hughesi & 8.9 & $7.8-8.1$ \\
\hline
\end{tabular}

Note: The letters " $T$ " and " $\mathrm{B}$ " signify the morphotypic top and bottom of each species' range. 
sent in virtually all Leg 115 sites and is only sparsely present in sites north of the equator (Site 714). Above this transition, in the late Miocene through Holocene, silica is common and radiolarians are well preserved.

In the Atlantic, this pattern is exactly reversed: biogenic silica is common and radiolarians are well preserved in the Neogene and Paleogene, prior to about $11 \mathrm{Ma}$. In the late Miocene through Pleistocene, however, silica is virtually absent throughout tropical and temperate latitudes (e.g., Riedel and Sanfilippo, 1970; Foreman, 1973; Johnson, 1977, 1983). This event seems to be of oceanwide significance and implies a major shift in the primary productivity and/or dissolution characteristics of much of the Atlantic.

Several oceanographic events of global significance occurred near the middle/late Miocene transition, including the expansion of the Antarctic ice cap (Shackleton and Kennett, 1975) and intensified flow in the Circumpolar Current (Johnson et al., 1983). Because the major wind-driven and thermohaline current systems are inextricably linked (Johnson, 1982), it is difficult to specify a particular triggering event to account for a number of closely occurring transitions observed in the depositional record. Several possibilities, however, may be considered.

1. The gradual closure of the Tethyan Seaway during the Neogene (Norton and Sclater, 1979) is one possible factor that may have been crucial in controlling the silica budgets of the Atlantic and Indian oceans, leading to preferential silica deposition in only one of the two oceans. It is beyond the scope of this paper, however, to suggest the particular threshold conditions and chemical parameters that might account for this striking shift in firstorder patterns of silica accumulation in the two oceans.

2. During the Neogene, as Australia migrated northward and Indonesian seaways closed, the Pacific and Indian oceans became less connected in tropical latitudes. One might expect that microfossil assemblages would reflect this closure and geographic separation of the two oceans. One piece of evidence in this direction is that the Eocene radiolarian assemblages of the two oceans are remarkably comparable, and the succession of events (Appendix C) in the Indian Ocean is essentially identical with that of the Pacific (Sanfilippo et al., 1985). Thus, there is no evidence of significantly diachronous events in the Paleogene. In the late Neogene, however, as the closure of the Indonesian seaways progressed, there is evidence for substantial diachroneity of events, up to several million years in some instances (Johnson and Nigrini, 1985).

An obvious strategy for testing this notion is to examine other microfossil groups in the tropical Indian and Pacific regions to see if there is a pattern of increasingly diachronous events toward the middle and late Neogene, with the gradual closure of Indonesian seaways.

\section{ACKNOWLEDGMENTS}

I thank my colleagues C. Nigrini, J.-P. Caulet, A. Sanfilippo, and W. Riedel for their ongoing discussions and suggestions in the intricacies of Cenozoic radiolarian biostratigraphy. This research was partially supported by a grant from the U.S. Science Advisory Committee of JOIDES through Texas A \& M University that allowed support for post-cruise investigations of sediment samples. I thank C. Nigrini, A. Sanfilippo, and L. Streeter for a careful review of the manuscript, and E. Evans for typing the tables. Partial support was also provided under NSF Grant No. OCE87-15956. This is Contribution No. 6935 of the Woods Hole Oceanographic Institution.

\section{REFERENCES}

Backman, J., Schneider, D., Rio, D., and Okada, H., in press. Neogene low latitude magnetostratigraphy from ODP Site 710 and revised age estimates of Miocene nannofossil datum events. Geology.

Baldauf, J. G., Thomas, E., Clement, B., Takayama, T., Weaver, P. E., Backman, J., Jenkins, G., Mudie, P. J., and Westberg-Smith, M. J., 1986. Magnetostratigraphic and biostratigraphic synthesis, Deep Sea Drilling Project Leg 94. In Ruddiman, W. F., Kidd, R. B., Thomas, E., et al., Init. Repts. DSDP, 94, Pt. 2: Washington (U.S. Govt. Printing Office), 1159-1205.

Barron, J. A., Nigrini, C. A., Pujos, A., Saito, T., Theyer, F., Thomas, E., and Weinreich, N., 1985. Synthesis of biostratigraphy, central equatorial Pacific, Deep Sea Drilling Project Leg 85: refinement of Oligocene to Quaternary biochronology. In Mayer, L., Theyer, F., Thomas, E., et al., Init. Repts. DSDP, 85: Washington (U.S. Govt. Printing Office), 905-934.

Berggren, W. A., Kent, D. V., and Van Couvering, J. A., 1985. The Neogene: Part 2, Neogene geochronology and chronostratigraphy. In Snelling, N. J. (Ed.), The Chronology of the Geological Record. Geol. Soc. Mem. (London), 10:211-260.

Bjorklund, K. R., and Goll, R. M., 1979. Internal skeletal structures of Collosphaera and Trisolenia: a case of repetitive evolution in the Collosphaeridae (Radiolaria). J. Paleontol., 53:1293-1326.

Caulet, J.-P., 1986. Radiolarians from the southwestern Pacific. In Kennett, J. P., von der Borch, C. C., et al., Init. Repts. DSDP, 90: Washington (U.S. Govt. Printing Office), 835-861.

Caulet, J.-P., and Nigrini, C. A., 1988. The genus Pterocorys (Radiolaria) from the tropical late Neogene of the Indian and Pacific Oceans. Micropaleontology, 34:217-235.

Foreman, H. P., 1973. Radiolaria of Leg 10 with systematics and ranges for the families Amphipyndacidae, Artostrobiidae, and Theoperidae. In Worzel, J. L., Bryant, W., et al., Init. Repts. DSDP, 10: Washington (U.S. Govt. Printing Office), 407-474.

Goll, R. M., 1980. Pliocene-Pleistocene radiolarians from the East $\mathrm{Pa}$ cific Rise and the Galapagos spreading center, Deep Sea Drilling Project Leg 54. In Rosendahl, B. R., Hekinian, R., et al., Init. Repts. DSDP, 54: Washington (U.S. Govt. Printing Office), 425453.

Hays, J. D., 1970. Stratigraphy and evolutionary trends of Radiolaria in North Pacific deep sea sediments. In Hays, J. D. (Ed.), Geological Investigations of the North Pacific. Mem. Geol. Soc. Am., 10:185218.

Johnson, D. A., 1977. Cenozoic radiolaria from the eastern tropical Atlantic, DSDP Leg 41. In Lancelot, Y., Seibold, E., et al., Init. Repts. DSDP, 41: Washington (U.S. Govt. Printing Office), 763789.

1982. Abyssal teleconnections: interactive dynamics of the deep ocean circulation. Paleogeogr., Paleoclimatol., Paleoecol., 38: 93-128.

1983. Cenozoic radiolarians from the Brazil Basin and Rio Grande Rise. In Barker, P. F., Carlson, R. L., Johnson, D. A., et al., Init. Repts. DSDP, 72: Washington (U.S. Govt. Printing Office), 783-791.

Johnson, D. A., Ledbetter, M. T., and Damuth, J. E., 1983. Neogene sedimentation and erosion in the Amirante Passage, western Indian Ocean. Deep Sea Res., 30:195-219.

Johnson, D. A., and Nigrini, C. A., 1985. Synchronous and time-transgressive Neogene radiolarian datum levels in the equatorial Indian and Pacific Oceans. Mar. Micropaleontol., 9:489-523.

Johnson, D. A., Schneider, D. A., Caulet, J.-P., Nigrini, C. A., and Kent, D. V., 1989. Pliocene-Pleistocene radiolarian events and magnetostratigraphic calibrations for the tropical Indian Ocean. Mar. Micropaleontol., 14:33-66.

Kling, S. A., 1971. Radiolaria. In Fischer, A. G., Heezen, B. C., et al., Init. Repts. DSDP, 6: Washington (U.S. Govt. Printing Office), 1069-1117,

Knoll, A. H., and Johnson, D. A., 1975. Late Pleistocene evolution of the collosphaerid radiolarian Buccinosphaera invaginata Haeckel. Micropaleontology, 21:60-68.

Lazarus, D. B., 1985. Evolution of the radiolarian species-complex Pterocanium: a preliminary survey. J. Paleontol., 59:183-220. 
Mato, C. Y., and Theyer, F., 1980. Lychnocanoma bandyca n. sp., a new stratigraphically important late Eocene radiolarian. In Sliter, W. V. (Ed.), Studies in Marine Micropaleontology and Paleoecology: A Memorial Volume to Orville L. Bandy. Spec. Publ., Cushman Found. Foraminiferal Res., 19:225-229.

Maurasse, F., and Glass, B., 1976. Radiolarian stratigraphy and North American microtektites in Caribbean core RC9-58: implications concerning late Eocene radiolarian chronology and the age of the Eocene/Oligocene boundary. Trans. Caribb. Geol. Conf., 7th, Guadeloupe, 1974, pp. 205-212.

Moore, T. C., 1972. Mid-Tertiary evolution of the radiolarian genus $\mathrm{Ca}$ locycletta. Micropaleontology, 18:144-152.

Nigrini, C., 1967. Radiolaria in pelagic sediments from the Indian and Atlantic Oceans. Bull. Scripps Inst. Oceanogr., 11:1-125.

, 1971. Radiolarian zones in the Quaternary of the equatorial Pacific Ocean. In Funnell, B. M., and Riedel, W. R. (Eds.), The Micropalaeontology of Oceans: Cambridge (Cambridge Univ. Press), 443-461.

1974. Cenozoic radiolaria from the Arabian Sea, DSDP Leg 23. In Davies, T., Luyendyk, B. P., et al., Init. Repts. DSDP, 26: Washington (U.S. Govt. Printing Office), 1051-1121. , 1977. Tropical Cenozoic Artostrobiidae (Radiolaria). Micropaleontology, 23:241-269.

1985. Radiolarian biostratigraphy in the central equatorial Pacific, Deep Sea Drilling Project Leg 85. In Mayer, L., Theyer, F., et al., Init. Repts. DSDP, 85: Washington (U.S. Govt. Printing Office), 511-551.

Nigrini, C., and Lombari, G., 1984. A Guide to Miocene Radiolaria. Spec. Publ., Cushman Found. Foraminiferal Res., No. 22.

Nigrini, C., and Caulet, J.-P., 1988. The genus Anthocyrtidium (Radiolaria) from the tropical late Neogene of the Indian and Pacific oceans. Micropaleontology, 34:341-360.

Norton, I. O., and Sclater, J. G., 1979. A model for the evolution of the Indian Ocean and the breakup of Gondwanaland. J. Geophys. Res., 84:6803-6830.

Riedel, W. R., and Sanfilippo, A., 1970. Radiolaria, Leg 4, Deep Sea Drilling Project. In Bader, R. G., Gerard, R. D., et al., Init. Repts. $D S D P$, 4: Washington (U.S. Govt. Printing Office), 503-575.

1971. Cenozoic Radiolaria from the western tropical Pacific, Leg 7. In Winterer, E. L., Riedel, W. R., et al., Init. Repts. DSDP, 7, Pt. 2: Washington (U.S. Govt. Printing Office), 1529-1672.

, 1978. Stratigraphy and evolution of tropical Cenozoic radiolarians. Micropaleontology, 24:61-96.

1986. Morphological characters for a natural classification of Cenozoic Radiolaria, reflecting phylogenies. Mar. Micropaleontol., 11:151-170.

Sanfilippo, A., Burckle, L. H., Martini, E., and Riedel, W. R., 1973. Radiolarians, diatoms, silicoflagellates, and calcareous nannofossils in the Mediterranean Neogene. Micropaleontology, 19:209-234.

Sanfilippo, A., and Riedel, W. R., 1973. Cenozoic Radiolaria (exclusive of theoperids, artostrobiids, and amphipyndacids) from the Gulf of Mexico, Deep Sea Drilling Project Leg 10. In Worzel, J. L., Bryant, W., et al., Init. Repts. DSDP, 10: Washington (U.S. Govt. Printing Office), 475-611.

1980. A revised generic and suprageneric classification of the artiscins (Radiolaria). J. Paleontol., 54:1008-1011.

1982. Revision of the radiolarian genera Theocotyle, Theocotylissa, and Thyrsocyrtis. Micropaleontology, 28:170-188.

Sanfilippo, A., Westberg-Smith, M. J., and Riedel, W. R., 1985. Cenozoic Radiolaria. In Bolli, H. M., Saunders, J. B., and Perch-Nielsen, K. (Eds.), Plankton Stratigraphy: Cambridge (Cambridge Univ. Press), 631-713.

Saunders, J. B., Bernoulli, D., Müller-Merz, E., Oberhänsli, H., PerchNielsen, K., Riedel, W. R., Sanfilippo, A., and Torrini, R., Jr., 1985. Stratigraphy of the late middle Eocene to early Oligocene in the Bath Cliff section, Barbados, West Indies. Micropaleontology, 30:390-425.

Shackleton, N. J., and Kennett, J. P., 1975. Paleotemperature history of the Cenozoic and the initiation of Antarctic glaciation: oxygen and carbon isotope analyses in DSDP Sites 277,279 , and 281 . In Kennett, J. P., Houtz, R. E., et al., Init. Repts. DSDP, 29: Washington (U.S. Govt. Printing Office), 743-755.
Streeter, L., 1988. Two species (one new) of Pliocene-Pleistocene Radiolaria from the central equatorial Indian Ocean. Micropaleontology, 34:63-66.

Westberg, M. J., and Riedel, W. R., 1982. Radiolaria from the Middle America Trench off Guatemala. In Aubouin, J., Von Huene, R., et al., Init. Repts. DSDP, 67: Washington (U.S. Govt. Printing Office), 401-424.

\section{Ms 115B-156}

Date of initial receipt: 4 January 1989

Date of acceptance: 13 November 1989

\section{APPENDIX A: SPECIES LIST}

Cited references present descriptions and illustrations of the concept of each species as applied in this study.

Acrobotrys tritubus Riedel; Nigrini and Lombari, 1984, p. N203, pl. 33, figs. 1a, $1 \mathrm{~b}$.

Amphirhopalum ypsilon Haeckel; Nigrini, 1971, p. 447, pl. 34, figs. $7 \mathrm{a}-7 \mathrm{c}$.

Anthocyrtidium angulare Nigrini; Nigrini, 1971, p. 445, pl. 34.1, figs. 3a, 3b.

Anthocyrtidium euryclathrum Nigrini and Caulet; Nigrini and Caulet, 1988 , p. 349 , pl. 1 , figs. $5-7$.

Anthocyrtidium jenghisi Streeter; Streeter, 1988, p. 63, pl. 1, figs. 1-4. Anthocyrtidium michelinae Caulet; Streeter, 1988, pp. 64-65, pl. 1, figs. 5-9; Nigrini and Caulet, 1988, p. 351, pl. 1, figs. 13, 14.

Anthocyrtidium pliocenica (Seguenza); Nigrini and Lombari, 1984, p. N149, pl. 27, figs. 2a, 2 b.

Artophormis barbadensis (Ehrenberg); Sanfilippo et al., 1985, p. 666, figs. 12.1a, 12.1b.

Artophormis gracilis Riedel; Sanfilippo et al., 1985, pp. 666-667, figs. $12.2 \mathrm{a}-12.2 \mathrm{c}$.

Buccinosphaera invaginata Haeckel; Knoll and Johnson, 1975, p. 63, pl. 1, figs. 3-7.

Calocyclas bandyca (Mato and Theyer); Mato and Theyer, 1980, p. 225, pl. 1, figs. 1-6; Saunders et al., 1985, pl. 5, figs. 1, 5-6.

Calocyclas hispida (Ehrenberg); Foreman, 1973, p. 434, pl. 9, fig. 18.

Calocyclas turris Ehrenberg; Riedel and Sanfilippo, 1978, p. 65, pl. 3, figs. 7,8 .

Calocycletta caepa Moore; Moore, 1972, p. 150, pl. 2, figs. 4-7; Nigrini and Lombari, 1984, p. N153, pl. 28, figs. 1a-1d.

Calocycletta costata (Riedel); Riedel and Sanfilippo, 1971, p. 1598, pl. $2 \mathrm{H}$, figs. $12-14$.

Calocycletta robusta Moore; Moore, 1972, p. 148, pl. 1, fig. 6.

Calocycletta serrata Moore; Moore, 1972, p. 148, pl. 2, fig. 1.

Calocycletta virginis Haeckel; Sanfilippo et al., 1985, p. 693, figs. 28.1a, 28.1b.

"Carpocanistrum" azyx Sanfilippo and Riedel; Sanfilippo and Riedel, 1973, p. 530, pl. 35, fig. 9; Sanfilippo et al., 1985, pp. 690-691, figs. 27.1a, 27.1b.

Carpocanopsis cristatum (Carnevale); Riedel and Sanfilippo, 1971, p. 1597, pl. 2G, figs. 1-7.

Collosphaera orthoconus (Haeckel); Bjorklund and Goll, 1979, p. 1317; Goll, 1980, pl. 1, figs. 10-11.

Collosphaera tuberosa Haeckel; Knoll and Johnson, 1975, p. 63, pl. 2, figs. 1-3.

Cryptoprora ornata Ehrenberg; Sanfilippo et al., 1985, p. 693, figs. $27.2 \mathrm{a}, 27.2 \mathrm{~b}$.

Cyrtocapsella cornuta Haeckel; Sanfilippo et al., 1985, p. 670, figs. $16.2 \mathrm{a}, 16.2 \mathrm{~b}$.

Cyrtocapsella tetrapera Haeckel; Sanfilippo et al., 1985, p. 670, figs. 16.1a, 16.1b.

Cyclampterium milowi Riedel and Sanfilippo; Riedel and Sanfilippo, 1971 , p. 1595 , pl. 3B, fig. 3.

Cyclampterium pegetrum Sanfilippo and Riedel; Riedel and Sanfilippo, 1971, pl. 2D, figs. 13-14.

Dendrospyris bursa Sanfilippo and Riedel; Sanfilippo et al., 1973, p. 217, pl. 2, figs. 9-13.

Diartus hughesi (Campbell and Clark); Sanfilippo et al., 1985, p. 655, fig. 8.11. 
Diartus petterssoni (Riedel and Sanfilippo); Sanfilippo et al., 1985, p. 657 , figs. $8.10 \mathrm{a}, 8.10 \mathrm{~b}$.

Dictyoprora mongolfieri (Ehrenberg); Sanfilippo et al., 1985, pp. 702703 , figs. 33.1a-33.1d.

Dictyoprora pirum (Ehrenberg); Nigrini, 1977, p. 251, pl. 4, fig. 8.

Didymocyrtis antepenultima Riedel and Sanfilippo; Sanfilippo et al., 1985 , p. 657 , fig. 8.6 .

Didymocyrtis avita (Riedel); Sanfilippo et al., 1985, pp. 657-658, fig. 8.8a.

Didymocyrtis laticonus (Riedel); Sanfilippo et al., 1985, p. 658, figs. $8.5 \mathrm{a}, 8.5 \mathrm{~b}$.

Didymocyrtis penultima (Riedel); Sanfilippo et al., 1985, p. 658, figs. $8.7 \mathrm{a}, 8.7 \mathrm{~b}$.

Didymocyrtis prismatica (Haeckel); Sanfilippo and Riedel, 1980, p. 1010; Riedel and Sanfilippo, 1971, pl. 2C, figs. 11-13, pl. 4, fig. 5.

Didymocyrtis tetrathalamus (Haeckel); Sanfilippo et al., 1985, p. 659; Nigrini, 1967, pl. 2, figs. 4a-4d.

Dorcadospyris alata (Riedel); Riedel and Sanfilippo, 1970, p. 523; Sanfilippo et al., 1985, pp. 661-662, fig. 10.7 .

Dorcadospyris ateuchus (Ehrenberg); Sanfilippo et al., 1985, p. 663, figs. $10.4 \mathrm{a}, 10.4 \mathrm{~b}$.

Dorcadospyris papilio (Riedel); Sanfilippo et al., 1985, pp. 663-664, figs. $10.2 \mathrm{a}, 10.2 \mathrm{~b}$

Eucyrtidium diaphanes Sanfilippo and Riedel; Sanfilippo et al., 1973, p. 221, pl. 5, figs. 12-14.

Eusyringium fistuligerum Ehrenberg; Sanfilippo et al., 1985, pp. 670671 , figs. 17.1a, 17.1b.

Eusyringium lagena (Ehrenberg); Sanfilippo et al., 1985, pp. 672-673, figs, 17.2a-17.2c.

Lamprocyrtis neoheteroporos Kling; Kling, 1973, p. 639, pl. 5, figs. 1718 ; pl. 15, figs. 4-5.

Lamprocyrtis nigriniae (Caulet); Sanfilippo et al., 1985, pp. 694-695, figs. 29.1a-29.1c.

Lamptonium fabaeforme chaunothorax Riedel and Sanfilippo; Riedel and Sanfilippo, 1970, p. 524, pl. 5, figs. 8, 9.

Lamptonium fabaeforme constrictum Riedel and Sanfilippo; Sanfilippo et al., 1985 , p. 674 , fig. 18.4 .

Lamptonium fabaeforme fabaeforme (Krasheninnikov); Sanfilippo et al., 1985 , p. 674 , fig. 18.2 .

Lithochytris vespertilio Ehrenberg; Riedel and Sanfilippo, 1970, p. 528, pl. 9, figs. 8,9 .

Lithocyclia angusta (Riedel); Sanfilippo et al., 1985, p. 653, figs. 7.3a7.3c.

Lithocyclia aristotelis (Ehrenberg) group; Sanfilippo et al., 1985, p. 653, figs. 7.2a-7.2d.

Lithocyclia crux Moore; Sanfilippo et al., 1985, p. 655, figs. 7.4a, 7.4b.

Lithocyclia ocellus Ehrenberg group; Sanfilippo et al., 1985, p. 655, figs. 7.1a, 7.1b; Riedel and Sanfilippo, 1986, pl. III, figs. 4-5.

Lithopera bacca Ehrenberg; Nigrini, 1967, p. 54, pl. 6, fig. 2.

Lithopera thornburgi Sanfilippo and Riedel; Sanfilippo and Riedel, 1970, p. 455 , pl. 2, figs. 4-6.

Lophocyrtis biaurita (Ehrenberg); Foreman, 1973, p. 442, pl. 8, figs. 23-26.

Lophocyrtis jacchia (Ehrenberg); Riedel and Sanfilippo, 1978, p. 70, pl. 7, fig. 1; Riedel and Sanfilippo, 1986, pl. VII, fig. 10.

Lychnocanoma bellum (Campbell and Clark); Foreman, 1973, p. 437, pl. 1, fig. 17; Riedel and Sanfilippo, 1986, pl. I, fig. 12.

Lychnocanoma elongata (Vinassa de Regny); Sanfilippo et al., 1985, p. 676 , figs. 19.1a, $19.1 \mathrm{~b}$.

Lychnodictyum audax Riedel; Sanfilippo et al., 1985, p. 677, fig. 21.2.

Periphaena tripyramis triangula (Sutton); Sanfilippo and Riedel, 1973, p. 523, pl. 9, figs. 10-11.

Phormocyrtis striata striata Brandt; Sanfilippo et al., 1985, p. 679, figs. 20.1a, 20.1b.

Phormostichoartus doliolum (Riedel and Sanfilippo); Nigrini, 1977, pp. 252-253, pl. 1, fig. 14.

Phormostichoartus fistula Nigrini; Nigrini, 1977, p. 253, pl. 1, figs. 1113.

Phormostichoartus marylandicus (Martin); Nigrini, 1977, p. 253, pl. 2, figs. 1-4.

Podocyrtis ampla Ehrenberg; Riedel and Sanfilippo, 1970, p. 533, pl. 12, figs. 7-8.

Podocyrtis chalara Riedel and Sanfilippo; Sanfilippo et al., 1985, p. 697 , fig. 30.11 .
Podocyrtis diamesa Riedel and Sanfilippo; Sanfilippo et al., 1985, p. 695, fig. 30.2 .

Podocyrtis dorus Sanfilippo and Riedel; Sanfilippo and Riedel, 1973, p. 531, pl. 35, figs. 12-14.

Podocyrtis fasciolata Nigrini; Sanfilippo et al., 1985, p. 697, fig. 30.7.

Podocyrtis goetheana (Haeckel); Sanfilippo et al., 1985, pp. 697-698, fig. 30.12

Podocyrtis mitra Ehrenberg; Sanfilippo et al., 1985, p. 698, fig. 30.10.

Podocyrtis sinuosa Ehrenberg; Sanfilippo et al., 1985, pp. 698-699, fig. 30.9.

Podocyrtis trachodes Riedel and Sanfilippo; Sanfilippo et al., 1985, p. 699, fig. 30.14 .

Pterocanium prismatium Riedel; Sanfilippo et al., 1985, pp. 679-680, figs. 21.1a, 21.1b.

Pterocorys campanula Haeckel; Caulet and Nigrini, 1988, p. 226, pl. 1, figs. 2-5.

Pterocorys hertwigii (Haeckel); Caulet and Nigrini, 1988, p. 228, pl. 1 , figs. 11,12 .

Sethochytris babylonis (Clark and Campbell) group; Nigrini, 1974, p. 1068, pl. 1G, figs. 9-14.

Sethochytris triconiscus Haeckel; Sanfilippo et al., 1985, pp. 680-681, figs. 22.1a-22.1d.

Siphostichartus corona (Haeckel); Nigrini, 1977, pp. 257-258, pl. 2, figs. 5-7; Riedel and Sanfilippo, 1986, pl. V, fig. 10.

Solenosphaera omnitubus Riedel and Sanfilippo; Sanfilippo et al., 1985, p. 651 , figs. $4.3 \mathrm{a}, 4.3 \mathrm{~b}$.

Spongaster berminghami (Campbell and Clark); Sanfilippo et al., 1985, pp. $660-661$, figs. $9.3 \mathrm{a}, 9.3 \mathrm{~b}$

Spongaster pentas Riedel and Sanfilippo; Sanfilippo et al., 1985, p. 661 , figs. 9.2a, $9.2 \mathrm{~b}$

Spongaster tetras Ehrenberg; Sanfilippo et al., 1985, p. 661, figs. 9.la9.1c.

Stichocorys delmontensis (Campbell and Clark); Sanfilippo et al., 1985, pp. 681-682, figs. 23.1a, 23.1b.

Stichocorys johnsoni Caulet; Caulet, 1986, p. 851, pl. 6, figs. 5, 6.

Stichocorys peregrina (Riedel); Sanfilippo et al., 1985, p. 682, fig. 23.2.

Stichocorys wolffii Haeckel; Sanfilippo et al., 1985, p. 682, figs. 23.3a, 23.3b.

Stylatractus universus Hays; Hays, 1970, p. 215, pl. 1, figs. 1, 2. This taxon has also been designated as Axoprunum angelinum (Campbell and Clark); see Sanfilippo et al., 1985, pp. 651-652, figs. 5.1a, 5.1b.

Theocorys spongoconus Kling; Kling, 1971, p. 1087, pl. 5, fig. 6.

Theocorythium trachelium (Ehrenberg); Nigrini, 1967, p. 79, pl. 8, fig. 2 ; pl. 9, fig. 2.

Theocorythium vetulum Nigrini; Nigrini, 1971, p. 447, pl. 34.1, figs. 6a-6b.

Theocotyle conica Foreman; Sanfilippo et al., 1985, p. 683, fig. 25.3.

Theocotyle cryptocephala (Ehrenberg); Sanfilippo and Riedel, 1982, p. 178, pl. 2, figs. 4-7; Sanfilippo et al., 1985, p. 685, figs. 25.2a, $25.2 \mathrm{~b}$.

Theocotyle venezuelensis Riedel and Sanfilippo; Sanfilippo and Riedel, 1982, p. 179, pl. 2, figs. 8-12; Sanfilippo et al., 1985, pp. 685-686, figs. $25.4 \mathrm{a}-25.4 \mathrm{c}$.

Theocotylissa ficus (Ehrenberg); Sanfilippo et al., 1985, p. 686, figs. $25.7 \mathrm{a}, 25.7 \mathrm{~b}$.

Theocyrtis annosa (Riedel); Riedel and Sanfilippo, 1970, p. 535, pl. 15, fig. 9 .

Theocyrtis tuberosa Riedel; Sanfilippo et al., 1985, pp. 701-702, figs. 32.1a-32.1d.

Thyrsocyrtis bromia Ehrenberg; Sanfilippo and Riedel, 1982, pp. $172-$ 173 , pl. 1, figs. 17-20.

Thyrsocyrtis hirsuta (Krasheninnikov); Sanfilippo et al., 1985, p. 687, fig. 26.2.

Thyrsocyrtis rhizodon Ehrenberg; Sanfilippo and Riedel, 1982, pp. 173-174, pl. 1, figs. 14-16.

Thyrsocyrtis robusta Riedel and Sanfilippo; Sanfilippo and Riedel, 1982, p. 174 , pl. 1, fig. 5 .

Thyrsocyrtis tensa Foreman; Sanfilippo and Riedel, 1982, p. 176, pl. 1, figs. 6-7.

Thyrsocyrtis tetracantha (Ehrenberg); Sanfilippo et al., 1985, p. 690 figs. $26.8 \mathrm{a}, 26.8 \mathrm{~b}$.

Thyrsocyrtis triacantha (Ehrenberg); Sanfilippo et al., 1985, p. 690, figs. 26.7a, 26.7b.

Tristylospyris triceros (Ehrenberg); Sanfilippo et al., 1985, pp. 665-666, figs, $10.3 \mathrm{a}, 10.3 \mathrm{~b}$. 
APPENDIX C. PALEOGENE RADIOLARIAN EVENTS IN HOLES 709C AND 711A.

APPENDIX B. LATE NEOGENE RADIOLARIAN EVENTS IN HOLES 709A AND 710A.

\begin{tabular}{|c|c|c|}
\hline Events & Hole $709 \mathrm{~A}$ & Hole $710 \mathrm{~A}$ \\
\hline [B B. invaginata] & $\begin{array}{l}1 \mathrm{H}-2(2.2) \text { to } \\
1 \mathrm{H}-4(5.2)\end{array}$ & - \\
\hline T S. universus & $\begin{array}{l}1 \mathrm{H}-2(2.2) \text { to } \\
1 \mathrm{H}-4(5.2)\end{array}$ & $\begin{array}{l}1 \mathrm{H}-2(2.2) \text { to } \\
1 \mathrm{H}-4(5.2)\end{array}$ \\
\hline [B C. tuberosa] & $\begin{array}{l}1 \mathrm{H}-2(2.2) \text { to } \\
1 \mathrm{H}-4(5.2)\end{array}$ & $\begin{array}{l}1 \mathrm{H}-2(2.2) \text { to } \\
1 \mathrm{H}-4(5.2)\end{array}$ \\
\hline B $P$. hertwigii & $\begin{array}{l}1 \mathrm{H}-4(5.2) \text { to } \\
1 \mathrm{H}-\mathrm{CC}(10.1)\end{array}$ & $\begin{array}{l}1 \mathrm{H}-4(5.2) \text { to } \\
1 \mathrm{H}-\mathrm{CC}(9.5)\end{array}$ \\
\hline [T $A$, angulare] & $\begin{array}{l}1 \mathrm{H}-\mathrm{CC}(10.1) \text { to } \\
2 \mathrm{H}-2(12.4)\end{array}$ & $\begin{array}{l}1 \mathrm{H}-\mathrm{CC}(9.5) \text { to } \\
2 \mathrm{H}-2(11.7)\end{array}$ \\
\hline B L. nigriniae & $\begin{array}{l}1 \mathrm{H}-\mathrm{CC}(10.1) \text { to } \\
2 \mathrm{H}-2(11.2)\end{array}$ & $\begin{array}{l}1 \mathrm{H}-\mathrm{CC}(9.5) \text { to } \\
2 \mathrm{H}-2(11.7)\end{array}$ \\
\hline T L. neoheteroporos & $\begin{array}{l}2 \mathrm{H}-2(12.4) \text { to } \\
2 \mathrm{H}-4(15.4)\end{array}$ & $\begin{array}{l}1 \mathrm{H}-\mathrm{CC}(9.5) \text { to } \\
2 \mathrm{H}-2(11.7)\end{array}$ \\
\hline T A. michelinae & $\begin{array}{l}2 \mathrm{H}-4(15.4) \text { to } \\
2 \mathrm{H}-\mathrm{CC}(19.7)\end{array}$ & $\begin{array}{l}2 \mathrm{H}-2(11.7) \text { to } \\
2 \mathrm{H}-2(14.7)\end{array}$ \\
\hline [T $P$ prismatium] & $\begin{array}{l}2 \mathrm{H}-\mathrm{CC}(19.7) \text { to } \\
3 \mathrm{H}-2(21.9)\end{array}$ & $\begin{array}{l}2 \mathrm{H}-4(14.7) \text { to } \\
2 \mathrm{H}-6(17.7)\end{array}$ \\
\hline B $A$. angulare & $\begin{array}{l}2 \mathrm{H}-\mathrm{CC}(19.7) \text { to } \\
3 \mathrm{H}-2(21.9)\end{array}$ & $\begin{array}{l}2 \mathrm{H}-4(14.7) \text { to } \\
2 \mathrm{H}-6(17.7)\end{array}$ \\
\hline T A. jenghisi & $\begin{array}{l}3 \mathrm{H}-2(21.9) \text { to } \\
3 \mathrm{H}-4(24.9)\end{array}$ & $\begin{array}{l}2 \mathrm{H}-\mathrm{CC}(19.2) \text { to } \\
2 \mathrm{H}-\mathrm{CC}(21.4)\end{array}$ \\
\hline B $T$. trachelium & $\begin{array}{l}3 \mathrm{H}-2(21.9) \text { to } \\
3 \mathrm{H}-4(24.9)\end{array}$ & $\begin{array}{l}2 \mathrm{H}-\mathrm{CC}(19.2) \text { to } \\
3 \mathrm{H}-2(21.4)\end{array}$ \\
\hline [T S. peregrina] & $\begin{array}{l}3 \mathrm{H}-6(27.9) \text { to } \\
3 \mathrm{H}-\mathrm{CC}(29.4)\end{array}$ & $\begin{array}{l}3 \mathrm{H}-2(21.4) \text { to } \\
3 \mathrm{H}-3(23.1)\end{array}$ \\
\hline T A. pliocenica & $\begin{array}{l}3 \mathrm{H}-\mathrm{CC}(29.4) \text { to } \\
4 \mathrm{H}-2(31.6)\end{array}$ & $\begin{array}{l}3 \mathrm{H}-3(23.1) \text { to } \\
3 \mathrm{H}-\mathrm{CC}(28.8)\end{array}$ \\
\hline T $P$. fistula & $\begin{array}{l}4 \mathrm{H}-2(31.6) \text { to } \\
4 \mathrm{H}-\mathrm{CC}(39.1)\end{array}$ & $\begin{array}{l}3 \mathrm{H}-3(23.1) \text { to } \\
3 \mathrm{H}-\mathrm{CC}(28.8)\end{array}$ \\
\hline T L. audax & $\begin{array}{l}4 \mathrm{H}-2(31.6) \text { to } \\
4 \mathrm{H}-\mathrm{CC}(39.1)\end{array}$ & $\begin{array}{l}3 \mathrm{H}-\mathrm{CC}(28.8) \text { to } \\
4 \mathrm{H}-2(31.0)\end{array}$ \\
\hline T $P$ doliolum & $\begin{array}{l}4 \mathrm{H}-\mathrm{CC}(39.1) \text { to } \\
6 \mathrm{H}-2(50.9)\end{array}$ & $\begin{array}{l}3 \mathrm{H}-\mathrm{CC}(28.8) \text { to } \\
4 \mathrm{H}-2(31.0)\end{array}$ \\
\hline B A. ypsilon & $\begin{array}{l}4 \mathrm{H}-\mathrm{CC}(39.1) \text { to } \\
6 \mathrm{H}-2(50.9)\end{array}$ & $\begin{array}{l}4 \mathrm{H}-2(31.0) \text { to } \\
4 \mathrm{H}-4(34.0)\end{array}$ \\
\hline$[S$. berminghami $\rightarrow S$. pentas] & - & - \\
\hline T $S$. delmontensis & $\begin{array}{l}6 \mathrm{H}-\mathrm{CC}(58.3) \text { to } \\
7 \mathrm{H}-4(63.5)\end{array}$ & $\begin{array}{l}4 \mathrm{H}-6(37.8) \text { to } \\
4 \mathrm{H}-\mathrm{CC}(38.3)\end{array}$ \\
\hline T S. omnitubus & $\begin{array}{l}7 \mathrm{H}-4(63.5) \text { to } \\
7 \mathrm{H}-6(66.5)\end{array}$ & $\begin{array}{l}4 \mathrm{H}-6(37.0) \text { to } \\
4 \mathrm{H}-\mathrm{CC}(38.3)\end{array}$ \\
\hline T S. corona & $\begin{array}{l}7 \mathrm{H}-4(63.5) \text { to } \\
7 \mathrm{H}-6(66.5)\end{array}$ & $\begin{array}{l}4 \mathrm{H}-\mathrm{CC}(38.3) \text { to } \\
6 \mathrm{H}-2(50.2)\end{array}$ \\
\hline T A. tritubus & $\begin{array}{l}7 \mathrm{H}-6(66.5) \text { to } \\
7 \mathrm{H}-\mathrm{CC}(68.0)\end{array}$ & $\begin{array}{l}4 \mathrm{H}-\mathrm{CC}(38.3) \text { to } \\
6 \mathrm{H}-2(50.2)\end{array}$ \\
\hline T S. johnsoni & - & $\begin{array}{l}4 \mathrm{H}-\mathrm{CC}(38.3) \text { to } \\
6 \mathrm{H}-2(50.2)\end{array}$ \\
\hline T D. bursa & $\begin{array}{l}10 \mathrm{H}-2(89.5) \text { to } \\
10 \mathrm{H}-4(92.5)\end{array}$ & $\begin{array}{l}6 \mathrm{H}-\mathrm{CC}(57.5 \text { to } \\
7 \mathrm{H}-2(59.7)\end{array}$ \\
\hline$[S$. delmontensis $\rightarrow S$. peregrina $]$ & $\begin{array}{l}10 \mathrm{H}-2(89.5) \text { to } \\
10 \mathrm{H}-4(92.5)\end{array}$ & $\begin{array}{l}7 \mathrm{H}-2(59.7) \text { to } \\
7 \mathrm{H}-4(62.7)\end{array}$ \\
\hline B $S$. omnitubus & $\begin{array}{l}10 \mathrm{H}-4(92.5) \text { to } \\
10 \mathrm{H}-\mathrm{CC}(96.9)\end{array}$ & $\begin{array}{l}7 \mathrm{H}-2(59.7) \text { to } \\
7 \mathrm{H}-4(62.7)\end{array}$ \\
\hline [T D. hughesi] & $\begin{array}{l}10 \mathrm{H}-\mathrm{CC}(96.9) \text { to } \\
11 \mathrm{H}-4(102.1)\end{array}$ & $\begin{array}{l}7 \mathrm{H}-4(62.7) \text { to } \\
7 \mathrm{H}-6(65.7)\end{array}$ \\
\hline B A. tritubus & $\begin{array}{l}11 \mathrm{H}-4(102.1) \text { to } \\
11 \mathrm{H}-\mathrm{CC}(106.6)\end{array}$ & $\begin{array}{l}7 \mathrm{H}-6(65.7) \text { to } \\
7 \mathrm{H}-\mathrm{CC}(67.1)\end{array}$ \\
\hline T $D$. petterssoni & - & $\begin{array}{l}8 \mathrm{H}-\mathrm{CC}(76.6) \text { to } \\
9 \mathrm{H}-2(78.8)\end{array}$ \\
\hline [D. petterssoni $\rightarrow$ D. hughesi] & - & $\begin{array}{l}8 \mathrm{H}-\mathrm{CC}(76.6) \text { to } \\
9 \mathrm{H}-2(78.8)\end{array}$ \\
\hline B D. hughesi & - & $\begin{array}{l}9 \mathrm{H}-2(78.8) \text { to } \\
9 \mathrm{H}-4(81.8)\end{array}$ \\
\hline
\end{tabular}

Notes: The letters "T" and "B" signify the morphotypic top and bottom of each species' range. Events in brackets, [ ], define zonal boundaries (Sanfilippo et al., 1985). Columns under hole numbers list the core and section numbers, and the sub-bottom depths (mbsf; in parentheses), between which each event occurs. Complete species names are given in the Species List (Appendix A).

\begin{tabular}{|c|c|c|}
\hline Events & Hole $709 \mathrm{C}$ & Hole 711A \\
\hline B $T$. spongoconus & $\begin{array}{l}29 X-2(268.6) \text { to } \\
29 X-4(271.6)\end{array}$ & - \\
\hline T D. mongolfieri & $\begin{array}{l}29 X-C C(276.1) \text { to } \\
30 X-2(278.3)\end{array}$ & $\begin{array}{l}16 \mathrm{X}-\mathrm{CC}(153.0) \text { to } \\
17 \mathrm{X}-2(155.2)\end{array}$ \\
\hline T S. babylonis & $\begin{array}{l}29 X-C C(276.1) \text { to } \\
30 X-2(278.3)\end{array}$ & $\begin{array}{l}16 \mathrm{X}-\mathrm{CC}(153.0) \text { to } \\
17 \mathrm{X}-2(155.2)\end{array}$ \\
\hline A. barbadensis $\rightarrow$ A. gracilis & $\begin{array}{l}29 \mathrm{X}-\mathrm{CC}(276.1) \text { to } \\
30 \mathrm{X}-2(278.3)\end{array}$ & $\begin{array}{l}16 \mathrm{X}-\mathrm{CC}(153.0) \text { to } \\
17 \mathrm{X}-2(155.2)\end{array}$ \\
\hline T L. bellum & $\begin{array}{l}29 \mathrm{X}-\mathrm{CC}(276.1) \text { to } \\
30 \mathrm{X}-2(278.3)\end{array}$ & $\begin{array}{l}16 \mathrm{X}-\mathrm{CC}(153.0) \text { to } \\
17 \mathrm{X}-2(155.2)\end{array}$ \\
\hline$[L$. aristotelis $\rightarrow$ L. angusta $]$ & $\begin{array}{l}29 \mathrm{X}-\mathrm{CC}(276.1) \text { to } \\
30 \mathrm{X}-2(278.3)\end{array}$ & $\begin{array}{l}16 \mathrm{X}-\mathrm{CC}(153.0) \text { to } \\
17 \mathrm{X}-2(155.2)\end{array}$ \\
\hline T L. jacchia & $\begin{array}{l}29 \mathrm{X}-\mathrm{CC}(276.1) \text { to } \\
30 \mathrm{X}-2(278.3)\end{array}$ & $\begin{array}{l}16 \mathrm{X}-\mathrm{CC}(153.0) \text { to } \\
17 \mathrm{X}-2(155.2)\end{array}$ \\
\hline T C. ornata & $\begin{array}{l}29 \mathrm{X}-\mathrm{CC}(276.1) \text { to } \\
30 \mathrm{X}-2(278.3)\end{array}$ & $\begin{array}{l}16 \mathrm{X}-\mathrm{CC}(153.0) \text { to } \\
17 \mathrm{X}-2(155.2)\end{array}$ \\
\hline B $T$. tuberosa & $\begin{array}{l}30 X-C C(285.8) \text { to } \\
31 X-2(288.0)\end{array}$ & $\begin{array}{l}17 X-4(158.2) \text { to } \\
17 X-6(161.2)\end{array}$ \\
\hline T C. bandyca & $\begin{array}{l}30 X-C C(285.8) \text { to } \\
31-2(288.0)\end{array}$ & $\begin{array}{l}17 X-C C(162.7) \text { to } \\
18 X-2(164.9)\end{array}$ \\
\hline T C. turris & $\begin{array}{l}31 X-2(288.0) \text { to } \\
31 X-4(291.0)\end{array}$ & $\begin{array}{l}17 X-C C(162.7) \text { to } \\
18 X-2(164.9)\end{array}$ \\
\hline T T. rhizodon & $\begin{array}{l}31 X-2(288.0) \text { to } \\
31 X-4(291.0)\end{array}$ & $\begin{array}{l}17 X-C C(162.7) \text { to } \\
18 X-2(164.9)\end{array}$ \\
\hline T T. bromia & $\begin{array}{l}31 X-4(291.0) \text { to } \\
31 X-C C(295.4)\end{array}$ & $\begin{array}{l}17 \mathrm{X}-\mathrm{CC}(162.7) \text { to } \\
18 \mathrm{X}-2(164.9)\end{array}$ \\
\hline [T T. tetracantha] & $\begin{array}{l}31 X-4(291.0) \text { to } \\
31 X-C C(295.4)\end{array}$ & $\begin{array}{l}17 \mathrm{X}-\mathrm{CC}(162.7) \text { to } \\
18 \mathrm{X}-2(164.9)\end{array}$ \\
\hline T C. $a z y x$ & $\begin{array}{l}31 X-4(291.0) \text { to } \\
31 X-C C(295.4)\end{array}$ & $\begin{array}{l}17 X-C C(162.7) \text { to } \\
18 X-2(164.9)\end{array}$ \\
\hline T E. fistuligerum & $\begin{array}{l}32 X-2(297.6) \text { to } \\
323 X-4(300.6)\end{array}$ & $\begin{array}{l}17 X-C C(162.7) \text { to } \\
18 X-2(164.9)\end{array}$ \\
\hline T C. hispida & $\begin{array}{l}32 X-2(297.6) \text { to } \\
32 X-4(300.6)\end{array}$ & $\begin{array}{l}18 X-4(167.9) \text { to } \\
18 X-C C(172.4)\end{array}$ \\
\hline T T. triacantha & $\begin{array}{l}32 X-4(297.6) \text { to } \\
32 X-6(300.6)\end{array}$ & $\begin{array}{l}18 X-4(167.9) \text { to } \\
18 X-C C(172.4)\end{array}$ \\
\hline T $P$ goetheana & $\begin{array}{l}33 X-2(307.3) \text { to } \\
33 X-4(310.3)\end{array}$ & $\begin{array}{l}18 \mathrm{X}-\mathrm{CC}(172.4) \text { to } \\
19 \mathrm{X}-2(174.6)\end{array}$ \\
\hline [B C. bandyca] & $\begin{array}{l}33 X-2(307.3) \text { to } \\
33 X-4(310.3)\end{array}$ & $\begin{array}{l}18 X-C C(172.4) \text { to } \\
19 X-2(174.6)\end{array}$ \\
\hline B C. turris & $\begin{array}{l}33 X-2(307.3) \text { to } \\
33 X-4(310.3)\end{array}$ & $\begin{array}{l}19 X-4(177.6) \text { to } \\
19 X-6(180.6)\end{array}$ \\
\hline [B C. $a z y x]$ & $\begin{array}{l}33 X-2(307.3) \text { to } \\
33 X-4(310.3)\end{array}$ & $\begin{array}{l}19 X-4(177.6) \text { to } \\
19 X-6(180.6)\end{array}$ \\
\hline T $T$. ficus & $\begin{array}{l}33 X-2(307.3) \text { to } \\
33 X-4(310.3)\end{array}$ & $\begin{array}{l}19 X-4(177.6) \text { to } \\
19 X-6(180.6)\end{array}$ \\
\hline B $T$, bromia & $\begin{array}{l}33 X-2(307.3) \text { to } \\
33 X-4(310.3)\end{array}$ & $\begin{array}{l}19 X-4(177.6) \text { to } \\
19 X-6(180.6)\end{array}$ \\
\hline T $P$ chalara & $\begin{array}{l}33 X-2(307.3) \text { to } \\
33 X-4(310.3)\end{array}$ & $\begin{array}{l}19 \mathrm{X}-6(180.6) \text { to } \\
19 \mathrm{X}-\mathrm{CC}(182.1)\end{array}$ \\
\hline B $T$. tetracantha & $\begin{array}{l}33 X-2(307.3) \text { to } \\
33 X-4(310.3)\end{array}$ & $\begin{array}{l}19 \mathrm{X}-6(180.6) \text { to } \\
19 \mathrm{X}-\mathrm{CC}(182.1)\end{array}$ \\
\hline B D. pirum & $\begin{array}{l}33 X-4(310.3) \text { to } \\
33 X-C C(314.8)\end{array}$ & $\begin{array}{l}19 X-4(177.6) \text { to } \\
19 X-6(180.6)\end{array}$ \\
\hline T S. triconiscus & $\begin{array}{l}33 \mathrm{X}-4(310.3) \text { to } \\
33 \mathrm{X}-\mathrm{CC}(314.8)\end{array}$ & $\begin{array}{l}19 X-6(180.6) \text { to } \\
19 X-C C(182.1)\end{array}$ \\
\hline L. ocellus $\rightarrow$ L. aristotelis & $\begin{array}{l}33 \mathrm{X}-4(310.3) \text { to } \\
33 \mathrm{X}-\mathrm{CC}(314.8)\end{array}$ & $\begin{array}{l}19 X-6(180.6) \text { to } \\
19 X-C C(182.1)\end{array}$ \\
\hline B $T$. triceros & $\begin{array}{l}33 \mathrm{X}-4(310.3) \text { to } \\
33 \mathrm{X}-\mathrm{CC}(314.8)\end{array}$ & $\begin{array}{l}19 X-6(180.6) \text { to } \\
19 X-C C(182.1)\end{array}$ \\
\hline [B $P$ goetheana] & $\begin{array}{l}33 X-4(310.3) \text { to } \\
33 X-C C(314.8)\end{array}$ & $\begin{array}{l}19 X-6(180.6) \text { to } \\
19 X-C C(182.1)\end{array}$ \\
\hline $\mathrm{T}$ L. vespertilio & $\begin{array}{l}33 X-4(310.3) \text { to } \\
33 X-C C(314.8)\end{array}$ & $\begin{array}{l}19 X-C C(182.1) \text { to } \\
20 X-2(184.3)\end{array}$ \\
\hline T P. trachodes & $\begin{array}{l}34 X-2(317.0) \text { to } \\
34 X-4(320.0)\end{array}$ & $\begin{array}{l}20 X-2(184.3) \text { to } \\
20 X-4(187.3)\end{array}$ \\
\hline T $P$ striata striata & $\begin{array}{l}34 X-2(317.0) \text { to } \\
34 X-4(320.0)\end{array}$ & $\begin{array}{l}20 X-2(184.3) \text { to } \\
20 X-4(187.3)\end{array}$ \\
\hline$[P$. mitra $\rightarrow P$ chalara $]$ & $\begin{array}{l}34 X-2(317.0) \text { to } \\
34 X-4(320.0)\end{array}$ & $\begin{array}{l}20 X-4(187.3) \text { to } \\
20 X-C C(191.7)\end{array}$ \\
\hline B C. ornata & $\begin{array}{l}33 X-2(307.3) \text { to } \\
33 X-4(310.3)\end{array}$ & $\begin{array}{l}20 X-4(187.3) \text { to } \\
20 X-C C(191.7)\end{array}$ \\
\hline T L. biaurita & $\begin{array}{l}35 X-2(326.6) \text { to } \\
35 X-4(329.6)\end{array}$ & $\begin{array}{l}21 X-4(196.9) \text { to } \\
21 X-C C(201.4)\end{array}$ \\
\hline T $P$ fasciolata & $\begin{array}{l}35 X-2(326.6) \text { to } \\
35 X-4(329.6)\end{array}$ & $\begin{array}{l}21 X-4(196.9) \text { to } \\
21 X-C C(201.4)\end{array}$ \\
\hline T E. lagena & $\begin{array}{l}35 \mathrm{X}-6(332.6) \text { to } \\
35 \mathrm{X}-\mathrm{CC}(334.1)\end{array}$ & $\begin{array}{l}22 X-4(206.6) \text { to } \\
22 X-C C(211.1)\end{array}$ \\
\hline
\end{tabular}


Appendix C (continued).

\begin{tabular}{|c|c|c|}
\hline Events & Hole $709 \mathrm{C}$ & Hole 711A \\
\hline B S. triconiscus & $\begin{array}{l}35 \mathrm{X}-\mathrm{CC}(334.1) \text { to } \\
36 \mathrm{X}-2(336.3)\end{array}$ & $\begin{array}{l}22 X-C C(211.1) \text { to } \\
23 X-2(213.3)\end{array}$ \\
\hline$[P$ sinuosa $\rightarrow P$ mitra $]$ & $\begin{array}{l}35 X-C C(334.1) \text { to } \\
36 X-2(336.3)\end{array}$ & $\begin{array}{l}22 X-C C(211.1) \text { to } \\
23 X-2(213.3)\end{array}$ \\
\hline B $P$ trachodes & $\begin{array}{l}36 \mathrm{X}-2(336.3) \text { to } \\
36 \mathrm{X}-\mathrm{CC}(343.9)\end{array}$ & $\begin{array}{l}22 \mathrm{X}-4(206.6) \text { to } \\
22 \mathrm{X}-\mathrm{CC}(211.1)\end{array}$ \\
\hline E. lagena $\rightarrow$ E. fistuligerum & $\begin{array}{l}36 \mathrm{X}-\mathrm{CC}(343.9) \text { to } \\
37 \mathrm{X}-2(346.1)\end{array}$ & $\begin{array}{l}23 X-2(213.3) \text { to } \\
23 X-4(216.3)\end{array}$ \\
\hline B $P$ fasciolata & $\begin{array}{l}36 \mathrm{X}-\mathrm{CC}(343.9) \text { to } \\
37 \mathrm{X}-2(346.1)\end{array}$ & $\begin{array}{l}23 X-2(213.3) \text { to } \\
23 X-4(216.3)\end{array}$ \\
\hline T $P$ dorus & $\begin{array}{l}37 X-4(349.1) \text { to } \\
37 X-6(352.1)\end{array}$ & $\begin{array}{l}23 X-6(219.3) \text { to } \\
23 X-C C(220.7)\end{array}$ \\
\hline T $T$. venezuelensis & $\begin{array}{l}37 X-6(352.1) \text { to } \\
23 X-6(219.3)\end{array}$ & $\begin{array}{l}23 X-4(216.3) \text { to } \\
37 \mathrm{X}-\mathrm{CC}(353.7)\end{array}$ \\
\hline$[P$ phyxis $\rightarrow$ P. ampla $]$ & - & - \\
\hline T. conica & $\begin{array}{l}37 X-6(352.1) \text { to } \\
37 X-C C(353.7)\end{array}$ & $\begin{array}{l}23 X-C C(220.7) \text { to } \\
24 X-2(222.9)\end{array}$ \\
\hline T $T$. hirsuta & - & $\begin{array}{l}24 X-C C(230.3) \text { to } \\
25 X-2(232.5)\end{array}$ \\
\hline T $T$. robusta & - & $\begin{array}{l}25 X-2(232.5) \text { to } \\
25 X-4(235.5)\end{array}$ \\
\hline T L. fabaeformae constrictum & - & $\begin{array}{l}25 X-4(235.5) \text { to } \\
25 X-C C(240.0)\end{array}$ \\
\hline T L. fabaeformae chaunothorax & - & $\begin{array}{l}25 X-4(235.5) \text { to } \\
25 X-C C(240.0)\end{array}$ \\
\hline T. tensa $\rightarrow T$. triacantha & - & $\begin{array}{l}25 X-4(235.5) \text { to } \\
25 X-C C(240.0)\end{array}$ \\
\hline [B E. lagena] & $25 X-C C \overline{(240.0)}$ & $25 X-4(235.5)$ to \\
\hline T L. fabaeformae fabaeformae & - & $\begin{array}{l}25 X-4(235.5) \text { to } \\
25 X-C C(240.0)\end{array}$ \\
\hline
\end{tabular}

Notes: The letters " $\mathrm{T}$ " and " $\mathrm{B}$ " signify the morphotypic top and bottom of each species' range. Events in brackets, [ ], define zonal boundaries (Saunders et al., 1985; Sanfilippo et al., 1985). Columns under hole numbers list the core and section numbers, and the sub-bottom depths (mbsf; in parentheses), between which each event occurs. Complete species names are given in the Species List (Appendix A). 